\title{
1 Convergent mutations in tissue-specific regulatory 2 regions reveal novel cancer drivers
}

3 Nasa Sinnott-Armstrong ${ }^{1}$, Jose A. Seoane ${ }^{1,2,3}$, Richard Sallari ${ }^{4}$, Jonathan K.

4 Pritchard $^{1,5}$, Christina Curtis ${ }^{1,2,3 \# *}$, Michael P. Snyder ${ }^{1 \# *}$

51 Department of Genetics, Stanford University School of Medicine, Stanford, California, 6 USA

7

82 Department of Medicine, Division of Oncology, Stanford University School of

9 Medicine, Stanford, CA

103 Stanford Cancer Institute, Stanford University School of Medicine, Stanford, CA

114 Axiotl Inc, Cleveland, OH

125 Department of Biology, Stanford University, Stanford, CA

13 *Correspondence: cncurtis@stanford.edu (lead contact) and mpsnyder@stanford.edu

14 \# These authors contributed equally 


\section{Abstract}

16 Although much effort has been devoted to identifying coding mutations across cancer

17 types, regulatory mutations remain poorly characterized. Here, we describe a

18 framework to identify non-coding drivers by aggregating mutations in cell-type specific

19 regulatory regions for each gene. Application of this approach to 2,634 patients across

2011 human cancer types identified 60 pan-cancer, 22 pan-breast and 192 cancer specific

21 candidate driver genes that were enriched for expression changes. Analysis of

22 high-throughput CRISPR knockout screens revealed large, cancer specific growth

23 effects for these genes, on par with coding mutations and exceeding that for promoter

24 mutations. Amongst the five candidate drivers selected for further analysis, four (IPO9,

25 MED8, PLEKHA6, and OXNAD1) were associated with survival across multiple cancer

26 types. These studies demonstrate the power of our cell-type aware, convergent

27 regulatory framework to define novel tissue specific cancer driver genes, considerably

28 expanding evidence of functional non-coding mutations in cancer. 


\section{Introduction}

30 To date, much effort has been devoted to the analysis of coding regions within the

31 human genome to define somatic alterations associated with tumor growth and

32 progression (Bailey et al., 2018; Lawrence et al., 2014; Zehir et al., 2017). While many

33 recurrent clonal coding mutations have been defined, non-coding elements (including

34 promoters and enhancers) implicated in malignancy have been far more elusive due to

35 the need for large cohorts with whole genome sequencing (WGS) data and new analytic

36 approaches. Indeed, attempts to locate regulatory elements enriched for functional

37 mutations (Araya et al., 2016; Feigin et al., 2017; Melton et al., 2015; Weinhold et al.,

38 2014; Zhu et al., 2020) have revealed only a handful of target genes, most of which are

39 associated with core promoter variants. An example is the canonical oncogene TERT,

40 where promoter mutations can induce c-Myc activation and telomeric immortalization

41 (Berger et al., 2012; Huang et al., 2013; Wu et al., 1999). However, the vast majority of

42 genes are regulated by promoters as well as proximal and distal enhancer elements

43 (Schmidt et al., 2010), suggesting that the latter may harbor as of yet undiscovered

44 mutations. Indeed, the long non-coding RNA (IncRNA) gene PVT1 was recently

45 identified as a tissue-specific tumor suppressor DNA boundary element that regulates

46 MYC transcription (Cho et al., 2018), demonstrating a role for regulatory sequences of

47 IncRNAs in malignancy. A recent paper by Rheinbay et al identified a small number

48 (4-5) of driver mutations when combining coding and non-coding genomic elements per

49 cancer genome. However, even in this most recent study, analyses suggest that

50 discovery of noncoding mutations and driver genes is far from complete (Rheinbay et

51 al., 2020).

53 The tissue-specific epigenomic landscape of a cell dictates its response to oncogenic

54 cues and influences the selection of somatic alterations during tumor initiation

55 (Lawrence et al., 2014; Lowdon and Wang, 2017; Sack et al., 2018). Accordingly, we

56 reasoned that tissue-specific annotations may increase the power and interpretability of

57 cancer driver gene discovery. As evidenced by their enrichment in genome-wide

58 association studies (GWAS), expression quantitative trait loci (eQTLs), and

59 cross-species conservation analyses, sequence alterations in regulatory elements are

60 associated with functional changes in the expression of downstream target genes and

61 disease phenotypes (Maurano et al., 2012; Schaub et al., 2012; Zhou et al., 2020).

62 Meanwhile, putative regulatory element mutations have been shown to affect cancer

63 driver gene expression in relevant tissues (Takeda et al., 2018; Zhang et al., 2018).

64 Therefore, the systematic analysis of regulatory variants within active elements of the 
65 corresponding cell type of origin may improve the power to detect non-coding cancer

66 associated mutations.

67

68 Here, we leverage these principles to develop a generalizable analytic framework to

69 characterize cell-type-specific regulatory landscapes and non-coding mutational burden

70 across 2,634 patients spanning 11 cancer types (Supplemental Table 1). We focused on

71 regulatory variants within active elements in the cell type of origin, defined by the

72 chromatin state of the corresponding enhancer or promoter. To increase the power to

73 detect disease-associated variants, we aggregated regulatory information across all

74 elements for each gene, similar to recent work examining the ESR1 locus in breast

75 cancer (Bailey et al., 2016) and prostate cancer (Sallari et al., 2017). Using this

76 approach, we found both known and novel recurrently mutated regulatory regions, the

77 majority of which were associated with dysregulated expression of nearby genes and

78 differential survival outcomes. In particular, we identify IPO9 as a novel regulatory driver

79 mutation in breast cancer. Using high-throughput CRISPR screen data across cancer

80 cell lines (Meyers et al., 2017), we demonstrate that genes harboring recurrent

81 regulatory mutations, including IPO9, GUK1, MED8, and OXNAD1, were associated

82 with larger in vitro growth effects on average than genes enriched for coding mutations.

83 Together, these results highlight the power of aggregating regulatory information and the

84 use of cell-type-aware models to define novel oncogenic drivers across diverse cancers.

\section{Results}

\section{Analytical Framework}

88 We reasoned that the power to discover novel regulatory regions as well as driver

89 genes would be improved by combining regulatory information for each gene,

90 analogous to burden tests aggregating exonic information for coding sequences (Figure

91 1A). In order to capture information relevant for each cancer type, we used cell type

92 specific epigenetic data available from the ENCODE and Roadmap Epigenome

93 projects. We estimated mutational enrichment within regulatory regions of each gene by

94 permutation testing (Methods). To implement this approach, we first linked the distal

95 enhancer elements defined by the Roadmap Epigenomics Consortium (Roadmap

96 Epigenomics Consortium et al., 2015) to each of the 18,729 GENCODE genes using

97 the correlation-based links from Roadmap (Figure 1B). Each distal element can be

98 assigned to one or more genes. To verify the quality of these enhancer-promoter links,

99 we counted the number of linked genes present at each enhancer element

100 (Supplemental Figure 1C). Each distal element linked to $~ 5$ genes on average,

101 consistent with other studies (Fishilevich et al., 2017). 
103 To assess the quality of our regulatory links, we next intersected these links with

104 chromatin states from the corresponding cell type, producing a canonical

105 enhancer-enriched distribution of regulatory activity (Supplemental Figure 1D,

106 Supplemental Table 2). We compared the chromatin state annotations within each

107 cancer type on each side of a regulatory link and discovered an enrichment of

108 repressed regulatory elements linked to repressed promoters and active regulatory

109 elements linked to active promoters, consistent with expectations of domain-level

110 activation (Rao et al., 2014) (Supplemental Figure 1E). These results indicate that both

111 chromatin states and enhancer-gene links are stable and high quality.

113 To evaluate mutational enrichment in regulatory regions of all genes, we used SNV and

114 indel calls from WGS data from the International Cancer Genome Consortium (ICGC)

115 focusing on 11 cancer types with a minimum of $n=90$ individuals and tissue matched

116 epigenetic data (Figure 1C, Supplemental Table 1). In addition, the breast cancer cohort

117 was sufficiently large to enable evaluation of the etiologically distinct Basal, Luminal,

118 and HER2+ subgroups (Nik-Zainal et al., 2016). The variants from each cohort were

119 normalized for regional patient mutation rate (Methods), chromatin state, and cancer

120 type, intersected with each gene's aggregated regulatory regions and evaluated for

121 mutational enrichment. Enrichment was assessed by permutation testing (as in (Sallari

122 et al., 2017); 5000+ iterations), where a matching background set of regulatory

123 elements were randomly assigned to each gene (maintaining mutation rate and

124 chromatin state) and the number of mutations scored (Methods).

125

Excess mutational burden in aggregate distal regulatory regions in breast cancer

128 We first evaluated this approach in a WGS dataset composed of 560 breast cancers

129 stratified by three major subtypes: Basal $(n=167)$, Luminal $(n=320)$, and HER2+ $(n=$

130 73) (Nik-Zainal et al., 2016). We performed enrichment tests on 57,534

131 FANTOM-derived promoters for 20,209 Ensembl-annotated genes, where promoters for

132 the same gene were concatenated when evaluating enrichments (Methods). Consistent

133 with previous results, we observed an enrichment in mutations in the shared promoter

134 of RMRP and CCDC107 across the individual breast cancer cohorts (Nik-Zainal et al.,

135 2016; Rheinbay et al., 2017). Combining p-values across the three breast cancer

136 subtypes via Fisher's method revealed enrichment of promoter mutation in TP53 and

137 CCDC107, as previously reported. When considering only active promoter elements, we

138 identify enrichments in WDR74, ZNF143, MFSD11, SRSF2, VMA21, CDC42BPB, and

139 TMEM189 (Supplemental Table 3). Thus, analysis of single regulatory elements reveals 
140 excess mutational burden in numerous previously identified drivers, as well as novel

141 candidate drivers.

143 We hypothesized that aggregating distal regulatory elements would yield increased 144 power to detect candidate driver genes. For each of the 18,729 GENCODE genes we 145 aggregated the promoter-interacting regulatory elements and tested for an excess or 146 overburdening of distal mutations. In order to resolve cell-type-specific effects, we 147 examined combinations of different chromatin states that represent the regulatory profile 148 of mammary epithelial cells (e.g. poised enhancers, active enhancers, promoters, 149 Supplemental Table 2). Using this approach, we identify 22 putative distal regulatory 150 driver genes with FDR $<10 \%$, spanning numerous regulatory states. These candidates 151 included known driver genes such as MSL3 (Leiserson et al., 2013) and HLE (Osborne 152 et al., 2010) (Supplemental Table 4). In addition, we found significant enrichment for 153 mutations in regulatory regions of 17 novel genes, most notably IPO9, which was 154 specifically enriched in enhancer marked chromatin (Figure 2C). Mutations in regulatory 155 regions of IPO9 were significantly overburdened in basal subtype tumors where 15 156 patients harbored 16 mutations, compared to an expectation of $\sim 3.6$ patients (4.2-fold 157 enrichment, permutation p-value < 3.2e-6, Methods). An additional 3 patients across the 158 other subgroups exhibited IPO9 mutations, bringing the total to 18 (Fisher combined, 159 FDR adjusted q-value across all three breast cancer subtypes $=0.068$ ). Additionally, 160 PYCR2 exhibited an excess of regulatory mutations (23 mutations across 22 patients, 161 q-value $=0.002)$ in active promoter \& strong enhancer (H3K4me3)-marked regions, as 162 did SDE2 (18 mutations across 17 patients, q-value $=0.023$ ), SRP9 (24 mutations in 23 163 patients, q-value $=0.02)$, and PLEKHA6 (22 mutations in 21 patients, q-value $=0.04$, 164 Supplemental Figure 2C). PYCR2 catalyzes the last step of proline synthesis from 165 glutamate in the mitochondrion (De Ingeniis et al., 2012); SDE2 is a telomere repair 166 gene implicated in cell cycle regulation (Jo et al., 2016); SRP9 binds and inhibits Alu 167 element translation (Chang et al., 1996); and PLEKHA6 is poorly characterized. Also of

168 note, luminal tumors comprise a heterogeneous group that can be stratified based on 169 genomic features (Rueda et al., 2019), hence it is not surprising that mutational 170 enrichment is weaker than observed in Basal and HER2+ tumors (Figure 2D).

172 We further evaluated mutational burden in topological domains from the progenitor 173 human mammary epithelial (HMEC) cells, the closest normal breast cell type with

174 comprehensive epigenomic data (Rao et al. 2014) and observed a significant 175 enrichment in promoter variants for the topological domain containing PLEKHA6 176 (Supplemental Figure 2D). The differences between the enhancer-gene linked 177 enrichments and topological domain enrichments is likely because many regulatory 178 regions in a given topological domain do not contribute globally to the expression of 
179 genes that reside within that domain (Degner et al., 2012; Gasperini et al., 2019;

180 Kasowski et al., 2013; Kilpinen et al., 2013; McVicker et al., 2013).

182 Identification of IPO9 as a putative breast cancer oncogene

184 We next sought to evaluate whether individuals with mutations in IPO9 regulatory 185 regions had altered IPO9 expression. IPO9 was highly expressed in MCF-7, which 186 contains a mutation in the IPO9 regulatory region, but not in HMEC cells, consistent 187 with its dysregulation in malignancy. In the independent METABRIC cohort, IPO9 188 expression was higher in Basal subtype tumors (Supplemental Figure 3A). Additionally, 189 IPO9 (1q32) is amplified in $26 \%$ of early stage breast cancers in the METABRIC cohort 190 and $22 \%$ of advanced breast cancers in the Metastatic Breast Cancer Project (Figure $1913 A)$. Among the 560 breast cancer patients with WGS data, only a subset $(n=268)$ had 192 matched RNA-seq data, four of which had IPO9 mutations. While underpowered to 193 detect an eQTL signal, IPO9 expression was higher in patients with IPO9 regulatory 194 mutations (Supplemental Figure 3B). In addition, when examining three validation 195 cohorts of whole genome sequenced tumors, we observed an additional 19 individuals 196 mutated in DNase regions of enhancer-marked chromatin at IPO9 (Figure 3B).

197 Collectively, these data suggest that increased IPO9 expression can occur through a 198 variety of mechanisms, including gene amplification, distal regulatory mutations, and 199 proximal mutations at the promoter, consistent with known oncogenes.

201 The epigenetic landscape of breast cancer surrounding the IPO9 locus is complex and 202 includes large open chromatin regions (defined using DNase-seq), actively transcribed 203 genes (RNA-seq), and regulatory elements (H3K27ac ChIP-seq; Figure 3C). Hi-C data 204 from HMEC cells (Rao et al. 2014) suggests that IPO9 lies at the boundary of two 205 topological domains, similar to that reported for other regulatory mutations in cancer 206 (Flavahan et al., 2016; Hnisz et al., 2016). We next examined individual regulatory 207 elements containing mutations. One such highly mutated element was located in an 208 intron of NAV1, approximately $50 \mathrm{~Kb}$ away from the IPO9 promoter and $120 \mathrm{~Kb}$ away 209 from the NAV1 promoter (Figure 3D). This element contains a CTCF binding site, active $210 \mathrm{H} 3 \mathrm{~K} 27 \mathrm{ac}$ and $\mathrm{H} 3 \mathrm{~K} 4 \mathrm{me} 1$ marks, as well as a number of conserved regions and DNase 211 hypersensitivity sites. Across all tumors with WGS, there were four breast cancer 212 patients each with a single mutation in this enhancer: one mutation located in a 213 conserved region $\sim 800$ bp away, a second located directly adjacent to the CTCF binding 214 site, and two more with mutations located in the DNase hypersensitivity site that is 215 associated with increased STAT3 and FOS binding upon estrogen stimulation in 216 MCF-10A cells (ENCODE Project Consortium, 2012). A similar trend was observed in 217 the IPO9 UTR, where four regulatory mutations were also present (Supplemental Figure 
218 3C). Together, these data implicate somatic alterations in IPO9 regulatory elements in 219 breast cancer pathogenesis, as further explored below.

220

221 Pan-cancer aggregate regulatory analysis discovers functional driver genes

223 We next expanded our analyses to catalogue pan-cancer regulatory driver mutations.

224 We first individually examined the same 20,209 genes used in the breast cancer

225 analysis. As a baseline, when considering all chromatin states rather than restricting to

226 active states, canonical non-coding variants in the TERT promoter were observed, as

227 previously reported (Horn et al., 2013; Huang et al., 2013; Vinagre et al., 2013).

228 Enrichment was even stronger when analyses were restricted to active promoters for

229 the cancer type of interest (28-fold versus 14.9-fold enriched). Therefore, for each

230 cancer type we examined the mutational enrichment in the TSS regions using the

231 corresponding active chromatin state information for that type of cancer (Methods). This

232 analysis revealed enrichment in the promoters of the canonical oncogenes BCL2, TP53,

233 TERT, and CXCR4. We also aggregated the enrichment information across cancer

234 types, which revealed an overlapping, but distinct, set of promoters, including those for

235 BTG1, CCL15, TERT, and TP53 (Supplemental Figure 4C). Thus aggregating promoter

236 mutations across cell types validates canonical driver genes, including TP53 and TERT.

238 We subsequently performed an aggregated distal regulatory element analysis, where

239 we initially employed a parametric approximation (Methods) and then validated

240 significant results with permutation testing. In contrast to methods that focus exclusively

241 on canonical promoter mutations, by aggregated distal regulatory state-specific

242 mutations, we identify numerous novel associations, including both cancer-specific $(\mathrm{n}=$

243 183) and pan-cancer $(n=40)$ mutated gene landscapes (Figure 4A, FDR of 10\%,

244 Supplemental Tables 5-6). For genes with at least one cancer-specific enrichment, we

245 quantified the significance across more than one cancer type via increasingly stringent

246 FDR cutoffs (Figure 4B).

247

248 One example of a hypermutated distal region was a segment associated with OXNAD1

249 and GALNT15, located 30kb apart. The aggregated distal regions for these genes were

250 specifically overburdened by mutations in CLL and melanoma (enrichment $=4.5$ and

251 1.63-fold, FDR-adjusted q-value $=0.058$ and 0.078), and OXNAD1 was previously

252 reported to be overburdened with promoter mutations in melanoma (Denisova et al.,

253 2015). Additionally, regulatory elements of the non-coding RNA transcript AC090953.1

254 located within an intron of GALNT15 was also overburdened with mutations (enrichment

$255=2.76$, q-value $=0.078$ ), though the enhancers overlap substantially with that of

256 OXNAD1 (Supplemental Table 7). Similar to germline expression QTLs (Tong et al., 
257 2017), co-regulation might mediate this shared enrichment signal. The TCERG1 gene 258 similarly harbored more mutations $(n=27)$ than expected by chance $(n=3.8 ; q<0.094)$ 259 across diverse cancer types, with enrichment in melanoma, esophageal, and ovarian

260 cancers. TCERG1 is a pro-apoptotic transcriptional elongation factor (Montes et al., 261 2015) implicated in cancer progression (Bailey et al., 2018; Forbes et al., 2017; Gao et 262 al., 2013) with two mutational hotspots in nearby coding regions of the gene 263 (Supplemental Figure 4G).

265 We further noted that the distribution of mutations varied significantly between promoter 266 and distal elements for putative drivers. For instance, OXNAD1 primarily harbored 267 promoter state mutations, whereas IFI16 and PYHIN1 share an enhancer element 268 (chr1:158968600-158969600) with mutations in 11 esophageal cancer patients 269 (Supplemental Table 8). Both of these sites would likely be detected with methods that 270 examine individual regulatory elements. However, other genes, such as BRCA1/NBR2 271 (Figure 4E) and $\mathrm{CDH} 13$ (Figure 4F), were overburdened with variants distributed across 272 multiple elements (e.g. promoters and distal elements), and hence would be overlooked 273 using conventional approaches, including those put forth in recent state-of-the-art single 274 element analyses (Rheinbay et al., 2020).

276 We further sought to evaluate whether our aggregated non-coding cell-type aware 277 driver discovery method can also recover known pan-cancer drivers of disease in 278 coding regions and UTRs. To this end, we focused on mutations in the "transcribed" 279 chromatin state, corresponding to active genes (Joshi and Struhl, 2005). After removing 280 genes for which the whole gene body lacked H3K36me3, and using Fisher's method to 281 combine p-values across cancer types, we confirmed the significant enrichment of 282 mutations in known driver genes TP53, BRAF, NRAS, SMAD4, and MUC3

283 (Supplemental Figure 4C, Supplemental Table 9-11, all but MUC3 reported in Rheinbay 284 et al., 2020). We also observed associations the UTR of NOTCH1 in CLL (Lobry et al., 285 2011) (4 patients, 48-fold enriched, $q<0.055$ ), and AHSA2 and USP34 in pediatric 286 brain cancers ( 7 and 6 patients, 20.5 -fold and 41 -fold enriched, $\mathrm{q}<0.0248$ and $\mathrm{q}<$ 287 0.0245). Overall, driver genes discovered using a cell type aware model overlapped 288 with those reported previously, but represent only a subset of those discovered using 289 aggregated noncoding elements, highlighting the power of our method to expand the 290 non-coding mutational landscape of cancer.

294 Our regulatory mutation analysis revealed a novel set of genes implicated in cancer. To 295 determine whether these genes are important for cell proliferation, we used 
296 genome-wide CRISPR screen data from Project Achilles (Meyers et al., 2017). These

297 analyses indicate that genes enriched for distal mutations tend to be highly deleterious

298 (Figure 5A). Although both distal- and promoter-mutated genes were enriched for

299 deleterious effects (Figure 5B, Supplemental Table 12), knockout of genes with distal

300 regulatory mutations had effects on cell growth comparable to coding mutations. Some

301 genes were essential in nearly all cancer cell lines, including MED8, GUK1, and SDE2

302 (Figure 5C), whereas others had cancer type specific growth effects (mostly

303 deleterious). For example, TMEM189 had severe growth defects in leukemia (intercept

$304-0.2$ across all lines; leukemia average $-0.52, p=0.038$, Supplemental Table 13) and

305 MAPK1 was less deleterious in myeloma and kidney cell lines (intercept -0.36 across all

306 lines; kidney average $0.044, p=0.049$ and myeloma average $0.12, p=0.038$,

307 Supplemental Table 14). Others were subtype specific - most notable was PAX5, where

308 the intercept across cell lines was $0.04(p=0.70)$, but in lymphoid neoplasms, the

309 regression effect was -0.40 ( $p=1.8 e-18$, Supplemental Table 15). In fact, putative

310 drivers were both more primary cancer type specific (Wilcoxon rank-sum test $\mathrm{W}=$

$311708190, p=0.037$ ) and had greater dependency scores (median dependency of -0.125

312 vs -0.06 , Wilcoxon rank-sum test $W=754210, p=0.009$ ) than other genes.

314 This suggests that the genes identified through aggregate regulatory mutation analysis

315 have strongly deleterious phenotypic consequences and confer selective advantages

316 through altered gene regulation commensurate with that of coding variants. While

317 strong pan-cancer tumor suppressor genes, such as PTEN and OXNAD1 (newly

318 discovered) (Supplemental Figure 5C), exhibited positive effects on growth, there were

319 very few regulatory genes with positive effects, whereas many genes, such as IPO9 and

320 the canonical oncogene MTOR, showed consistent negative growth effects across all

321 cell lines in Avana (Figure 5D).

\section{Fine-mapping at the IPO9 locus implicates RNA splicing and processing}

325 IPO9 knockouts exhibited dramatically reduced proliferation and this gene was

326 pan-essential in both the GeCKO and Avana screens. Indeed, the effect of IPO9

327 knockout on proliferation was far larger than other genes in the region (Figure 6A) and

328 persisted across cell types in the independent GeCKO screens (Supplemental Figure

329 6A). A similar decrease in proliferation was noted for TIMM17A in pleural and upper

330 digestive cancers (Supplemental Figure 6B).

332 This essentiality is further supported by the ExAC database (Lek et al., 2016), where

333 there was a significant depletion of missense variants $(z=3.11)$ in IPO9 and the

334 germline probability of loss of function intolerance (pLI) was 1.0. Motivated by this 
335 observation, we looked for rare cancer-associated regulatory variants at the locus using

336 the Oxford Brain Imaging Genetics Server (Elliott et al., 2018), and found a variant,

337 rs150641471, in an intron of NAV1 50kb from the IPO9 promoter, which was associated

338 with malignant thyroid neoplasm $(O R=1.05, p=3 e-22)$, diffuse large cell lymphoma

$339(\mathrm{OR}=1.1, \mathrm{p}=6.6 \mathrm{e}-12)$, and leukaemia $(\mathrm{OR}=1.004, \mathrm{p}=2.2 \mathrm{e}-6)$. This is consistent with

340 transposon screens in mice, which have implicated IPO9 in hematopoietic malignancy

341 (Guo et al., 2016).

343 To further characterize the role of IPO9 in cancer progression, we correlated the

344 gene-level growth effects for IPO9 with all other genes (Figure 5G-H) following

345 normalization, as previously described (Boyle et al., 2018) (Methods, Supplemental

346 Table 16). Gene ontology (GO) analysis of the 168 genes for which proliferation across

347 cell lines had a correlation greater than 0.3 with IPO9 revealed the striking enrichment

348 of non-coding RNA metabolic processes (7.29-fold, FDR adjusted $q=7.55 e-16$,

349 Supplemental Table 6) and catalytic activity on RNA (5.32-fold, q = 1.02e-4). Meanwhile,

350 the most negatively correlated genes include those involved in mRNA splicing via

351 transesterification (4.24-fold enriched in 1000 most negatively correlated genes, $q=$

352 1.36e-16; Figure 5I). These results implicate IPO9 in RNA splicing and processing.

\section{Recurrently mutated regulatory regions are associated with patient outcomes}

356 Since mutations in regulatory regions often result in gene expression changes, we next examined the association between the expression of genes with recurrently mutated regulatory regions and clinical outcome. We evaluated the specificity of survival associations across 27 cancer types with sufficient clinical information and follow-up duration from the TCGA Pan-Cancer Atlas, the largest compendium of cancer genomes that did not overlap with our non-TCGA ICGC discovery cohort (Bailey et al., 2018; Liu et al., 2018) (Supplemental Figure 6G). In order to limit the number of hypotheses tested, we only evaluated the association between IPO9, MED8, OXNAD1, PLEKHA6, and GUK1 expression and survival. While the trends varied between cancer types,

365 IPO9 (expression-increasing, risk-increasing), MED8 (expression-increasing, risk-increasing), and OXNAD1 (expression-increasing, risk-decreasing) were associated

367 with survival across multiple cancer types (Figure 7A-D, Supplemental Tables 17-18, 368 Supplemental Figure 7F,H-I, after adjusting for key clinical covariates and copy number at that locus, Methods). In addition, increased PLEKHA6 expression was protective in

370 bladder cancer and lung squamous cell cancer, and risk-increasing in clear cell renal 371 cell cancer. 
373 Next, we sought to evaluate cell-type specific driver effects and their prognostic

374 associations. We initially focused on breast cancer, given the large sample size and

375 long-term clinical follow-up available in the METABRIC cohort (Curtis et al., 2012;

376 Rueda et al., 2019). IPO9 expression was significantly associated with relapse free

377 survival (RFS) in Kaplan Meier analysis $(p<0.0001)$ and remained significant in a Cox

378 proportional hazard analysis adjusted for age, tumor grade and size, subtype, and copy

379 number $(H R=1.31$ [1.03, 1.7], $p=0.027$, Supplemental Figure 7D, Supplemental

380 Tables 19-22) (Methods). We further evaluated this association after stratifying for

381 breast cancer subgroups, revealing an even more striking relationship between IPO9

382 expression and relapse-free survival in luminal breast cancers ( $H R=1.80$ [1.29, 2.51], $p$

$383<0.001$, Figure 7E, Supplemental Figure 7E, Supplemental Tables 23-26).

385 Encouraged by this result, we evaluated the association between the expression of all

386 genes $(n=50)$ harboring recurrent regulatory or coding mutations from TCGA and

387 outcome in the METABRIC breast cancer cohort, for. A clear inflation of $p$-values is

388 noted, suggesting a number of genes are associated with survival. In the METABRIC

389 cohort (Supplemental Figure 7F-H), IPO9 was the fourth most significant gene, with

390 SDE2, which also exhibited large CRISPR growth effects, being the most significant

391 distal association. Of note, IPO9 expression was most strongly associated with relapse

392 free survival in luminal cases (Figure 7F). The distribution was similar for overall

393 survival, disease specific survival, and distant relapse (Supplemental Figure 7A-C).

394 These findings indicate that genes harboring recurrent regulatory mutations are

395 associated with patient prognosis, cementing their relevance in human cancers.

\section{Discussion}

399 Here we present a powerful framework to identify non-coding cancer driver genes

400 based on two key principles: aggregation of cell type specific regulatory elements and

401 cell type specific activity to identify novel non-coding driver gene mutations across

402 diverse cancer types. This approach defines driver mutations in multiple regulatory

403 elements simultaneously. Indeed, many regions and associated genes were not

404 identified previously. We demonstrate that mutations in the promoter of OXNAD1 are

405 likely oncogenic, consistent with previous claims (Denisova et al., 2015). Further, we

406 identify a IPO9, a nuclear actin transporter, implicated in mRNA metabolism and

407 alternative splicing, as a putative oncogene in breast cancer, melanoma, bladder

408 cancer, and mesothelioma. In addition to IPO9, other newly identified regulatory driver

409 genes, including SRSF2 and TCERG1, also modulate alternative splicing (Koedoot et

410 al., 2019; Montes et al., 2015; Pearson et al., 2008), suggesting a shared functional 
411 basis for these enrichments, similar to that also seen for alternative splicing in coding

412 mutations (Watson et al., 2013).

414 Previous work has implicated IPO9 in nuclear actin remodeling and adherence of

415 keratinocytes (Sharili et al., 2016), as well as in transcriptional control (Dopie et al.,

416 2012) and interferon signaling (Matsumiya et al., 2013). More recently, nuclear actin has

417 been implicated in the transport of homologous recombination double stranded breaks

418 to the periphery, where they can be efficiently repaired (Caridi et al., 2018). In addition,

419 nuclear actin dynamics, mediated by IPO9 and XPO6, have the potential to modulate

420 mRNA splicing through disruption of SMN2 (Viita et al., 2019). Alternative splicing and

421 other co-transcriptional metabolic processes acting on RNA are important for cancer

422 development (David and Manley, 2010; Koedoot et al., 2019), suggesting a multitude of

423 direct targets in promoting the hallmarks of cancer (Hanahan and Weinberg, 2011).

424 These diverse roles of nuclear actin in cellular proliferation and transcription are

425 consistent with our findings of mutational enrichment in IPO9 regulatory regions and the

426 association between elevated expression of IPO9 and shorter relapse-free and overall

427 survival in multiple cancer types. Together, this motivates further investigation of the

428 mechanism and diversity of nuclear actin as a class of oncogenes using high-content

429 imaging platforms with drug libraries and/or CRISPR tools.

More broadly, our method has uncovered a unique set of recurrently mutated genes not identified through conventional means, including recent large-scale non-coding analyses (Rheinbay et al., 2020). The observation that aggregated regulatory signals harbor enrichment not evident from the analysis of individual elements is reminiscent of progress in exome testing. Initial studies first evaluated individual coding variants, and later found increased power in gene-level burden tests. This suggests that applying novel approaches to the analysis of non-coding regions, including the development of specific driver detection tools, is of value.

440 The strong growth phenotypes of these genes identified via CRISPR/Cas9 screens 441 suggests that they might be constrained for coding variation, and that distal regulatory 442 elements with slight expression-altering mutations might jointly control expression at 443 multiple loci, akin to polygenic models in genome-wide association studies. These 444 findings also highlight the power of large scale genetic screens to inform driver gene 445 discovery and we identify an excess number of mutated genes with large deleterious 446 growth effects. It is worth noting, however, that loss of large-effect tumor suppressors 447 during serial passaging is anticipated, and such genes would not be identified in this 448 analysis. Finally, we illustrate how loss of function genetic screens can be used to fine 
449 map causal genes, evaluate cancer type specificity and determine functional

450 mechanisms, including direct annotation of pathways.

452 In sum, we present a general approach to identify regulatory regions enriched for

453 mutations while simultaneously correcting for background mutation rates. The

454 application of this approach to WGS data from 11 cancer types, lead to the identification

455 of multiple novel non-coding driver genes, supported by orthogonal validation of their

456 pan-cancer growth effects and prognostic associations. Of note, these findings likely

457 represent just the beginning, and we anticipate that additional non-coding drivers will be

458 identified through the application of this new cell-type aware, analytic framework to the

459 increasing number of WGS cancer datasets being generated with implications for

460 personal genome interpretation and prognosis. Together, we believe that improved

461 methods like these, as well as additional genomic and other omics data, will begin a

462 new large-scale effort to discover and interrogate regulatory drivers in cancer.

\section{Acknowledgements}

465 N.S.-A. is supported by the Department of Defense through a National Defense Science and 466 Engineering Grant and by a Stanford Graduate Fellowship. This work was supported by grants 467 from the NIH ENCODE/Production Center for Mapping Regulatory Regions of the Human 468 Genome - 3U54-HG006996-04S1 (M.S.), CEGS/Center for Personal Dynamic Regulomes 469 2RM1HG007735-06 (M.S., C.C.), the NIH Director's Pioneer Award - DP1-CA238296 (C.C.)

470 and the NCl Cancer Target Discovery and Development Network - U01 CA217851 (C. C.). This 471 work made use of resources provided by the Genetic and Bioinformatic Service Center at 472 Stanford University School of Medicine. We thank C. Suarez for feedback and tumor epigenome 473 matching and M. Wainberg, A. Sanghi, D. Rothschild, C. Smith, H. Ollila, J. Reuter, J. Gruber, 474 M. Pryzbilla and other members of the Curtis, Pritchard and Snyder labs for feedback on the 475 manuscript. This study makes use of patient data from the ICGC, TCGA, and METABRIC 476 studies, as well as epigenetic data from the ENCODE and Roadmap Epigenomics Project and 477 CRISPR screen data from DepMap. We thank all the individuals who contributed to the creation 478 of these resources, and particularly the patients whose biological samples form the basis of this 479 work. This study was performed on the traditional and unceded lands of the Muwekma Ohlone 480 people, and we are grateful for the opportunity to live and work here.

\section{Author contributions}

483 Conceptualization: N.S.-A. and R.S.

484 Methodology: N.S.-A., R.S., C.C., and M.P.S.

485 Software, Analysis, and Validation: N.S.-A. and J.A.S.

486 Investigation: N.S.-A., J.A.S., C.C. and M.P.S.

487 Writing and Editing: N.S.-A., J.K.P., C.C. and M.P.S.

488 Supervision: C.C. and M.P.S. 


\section{Competing interests}

491 C.C. is a scientific advisor to GRAIL and reports stock options, as well as consulting for GRAIL

492 and Genentech. M.P.S. is a co-founder and SAB member of Personalis.

\section{Data \& Code Availability}

495 This study makes use of patient data from the ICGC, TCGA, and METABRIC studies, as well as

496 epigenetic data from the ENCODE and Roadmap Epigenomics Project. Data from TCGA are

497 available publicly through the PanCan Atlas portal

498 (https://gdc.cancer.gov/about-data/publications/pancanatlas) and via application to dbGaP

499 accession phs000178.v1.p1. Data from ICGC are available on the ICGC website

500 (http://icgc.org/). Data from ENCODE and Roadmap are available on the ENCODE website

501 (http://encodeproject.org). Data from DepMap are available on the DepMap website

502 (https://depmap.org/portal/download/). Data from METABRIC are available at the European

503 Genotype-Phenotype Archive under Accession number EGAS00000000083 and as

504 supplementary tables in the current publication (Rueda et al., 2019).

506 The code needed to implement the methods described in this paper will be published along with 507 the accepted manuscript.

\section{Supplemental Tables}

509 Supplemental Table 1: Tumor samples included in the discovery cohort. The list of all

510 tumors used for initial discovery of driver mutations, including the aggregated tumor type used

511 for these analyses, the original cohort from ICGC, and the donor ID. Cancer type, cohort name,

512 and donor ID are listed.

514 Supplemental Table 2: Chromatin state definitions. The abbreviated names, equation (used

515 internally for specifying the definition), chromatin states, and DNase status of aggregated active

516 chromatin used for the analysis.

518 Supplemental Table 3: BRCA combined putative driver list. List of all putative driver genes

519 discovered in breast cancer using the fisher-combined p-values across cohorts, including the

520 chromatin state tested; resolution of tile resampling employed; mutation rate window; set of

521 chromatin loops evaluated; and expected mutation count across permutations, number of

522 observed mutations, and likewise for number of patients mutated, as well as the empirical

523 p-value and FDR-adjusted q-value. Only genes with a patient q-value $<0.1$ are reported.

524

525 Supplemental Table 4: BRCA combined active promoter and all promoter genes. List of all

526 genes putatively enriched in promoter mutations, either including all chromatin states or only

527 promoter chromatin annotations (active). 
529 Supplemental Table 5: Single-cancer coding driver genes. List of all genes putatively

530 enriched in coding mutations in each single cohort. Mutation, number of mutations observed;

531 patient, number of patients with mutations; permutations, number of permutations run to

532 evaluate significance; mean_mutation, average number of mutations in permutations;

533 mean_patient, average number of patients mutated in permutations; gtmutation, number of

534 permutations with mutation count exceeding the observed; gtpatient, number of permutations

535 with patient count exceeding the observed; p.pt, empirical p-value of patient mutations; q.pt

536 empirical FDR-adjusted p-value of patient mutations.

537

538 Supplemental Table 6: Pan-cancer combined coding drivers. List of all putative coding genes

539 discovered in the pan-cancer analysis using the fisher-combined p-values across cohorts. FDR

540 cutoff of $10 \%$ was used to report genes, and each gene was assessed using the

541 permutation-based approach.

542

543 Supplemental Table 7: Pan-cancer combined coding active drivers. List of all putative coding

544 genes discovered in the pan-cancer analysis using the fisher-combined p-values across

545 cohorts, but only using mutations located in actively transcribed regions. An FDR cutoff of $10 \%$

546 was used to report genes, and each gene was assessed using the permutation-based

547 approach.

548

549 Supplemental Table 8: Parametric single-cancer putative drivers. List of all putative

550 single-cancer aggregate regulatory drivers discovered using the parametric models. Cancer,

551 cancer type; links, regulatory element links used; state, chromatin state tested; rmr, window size

552 (bp) for calculating regional mutation rate; mutated, number of mutations observed, mean,

553 number of mutations expected; z, z-score based test statistic; log10pois, log of the p-value for

554 the poisson test; log10chi, log of the p-value for the chi squared test; log10z, log of the test

555 statistic for the $Z$ test; qchi, FDR-adjusted q-value for the chi square test; qpois, FDR-adjusted

556 q-value for the poisson test; qz, FDR-adjusted q-value for the $z$ test.

558 Supplemental Table 9: Pan-cancer combined putative drivers. List of all putative driver genes discovered in the pan-cancer analysis using the fisher-combined permutation p-values across

560 cohorts. Only genes that were validated with the permutation-based approach are reported.

561 State, chromatin state tested; mutations, number of observed mutations; patients, number of

562 mutated patients. QC is marked "FAIL" for histone, immunoglobulin, and RNA genes excluded

563 from downstream analysis.

565 Supplemental Table 10: OXNAD1/GALNT15 MELA mutated elements. List of mutations from

566 the linked regulatory regions of OXNAD1, GALNT15, and the nearby non-coding RNA. Each

567 row represents a mutation-gene combination, with the corresponding chromatin state and

568 regulatory region annotated. 
570 Supplemental Table 11: Mutations in a PYHIN1-IFI16 shared enhancer. List of individual

571 mutations located in the enhancer element shared by PYHIN1 and IFI16 across the esophageal

572 cancer cohort.

573

574 Supplemental Table 12: Essentiality comparison across genes. The fraction of gene effects

575 labeled essential for genes associated with coding mutations from TCGA (Bailey et al., 2018);

576 coding, promoter, enhancer, or UTR mutations from PCAWG (Rheinbay et al., 2020); and

577 aggregated regulatory regions in either breast cancer or the pan-cancer cohort (this study).

578

579 Supplemental Table 13: Cancer type specificity of TMEM189. Regression specification for the

580 cancer type specificity of TMEM189, adjusted for olfactory gene essentiality principal

581 components 1-5; gender; and source.

582

583

Supplemental Table 14: Cancer type specificity of MAPK1. Regression specification for the cancer type specificity of MAPK1, adjusted for olfactory gene essentiality principal components 1-5; gender; and source.

Supplemental Table 15: Cancer subtype specificity of PAX5. Regression specification for the cancer type specificity of PAX5, adjusted for olfactory gene essentiality principal components 1-5; cancer type; gender; and source.

590

Supplemental Table 16: Essentiality correlation with IP09. Table of pairwise batch-corrected correlations between each of the genes evaluated in the Avana screen and IPO9 across all 485 cell lines in the Avana dataset. types in the PanCanAtlas, the hazard ratio of expression changes for each of the five genes we

Supplemental Table 18: TCGA combined hazard ratios across cancer types. Combined hazard ratio for the five genes evaluated in multiple cancer types with adequate sample size.

602 Supplemental Table 19: Overall survival hazard ratios in METABRIC. Hazard ratios, for each

603 putative breast cancer driver gene, of expression against overall survival when adjusted for

604 standard clinical covariates.

606 Supplemental Table 20: Disease specific survival hazard ratios in METABRIC. Hazard ratios, 607 for each putative breast cancer driver gene, of expression against disease specific survival 608 when adjusted for standard clinical covariates.

610 Supplemental Table 21: Relapse free survival hazard ratios in METABRIC. Hazard ratios, for 611 each putative breast cancer driver gene, of expression against relapse free survival when

612 adjusted for standard clinical covariates. 
614 Supplemental Table 22: Disease and relapse free survival hazard ratios in METABRIC.

615 Hazard ratios, for each putative breast cancer driver gene, of expression against disease- and 616 relapse-free survival when adjusted for standard clinical covariates.

618 Supplemental Table 23: Overall survival hazard ratios in METABRIC, luminal cases only.

619 Hazard ratios, for each putative breast cancer driver gene, of expression against overall survival

620 when adjusted for standard clinical covariates, among luminal cases only. cases only. Hazard ratios, for each putative breast cancer driver gene, of expression against disease specific survival when adjusted for standard clinical covariates, among luminal cases 625 only. only. Hazard ratios, for each putative breast cancer driver gene, of expression against relapse

Supplemental Table 26: Disease and relapse free survival hazard ratios in METABRIC, against disease- and relapse-free survival when adjusted for standard clinical covariates,

\section{${ }_{635}$ Methods}

\section{Variant calls and sample inclusion}

638 Tumor types with whole genome sequencing as part of the International Cancer Genome 639 Consortium for which a minimum of 90 individuals were profiled and for whom matched 640 epigenomic data was available from the ENCODE and RoadMap Epigenome projects were 641 selected for inclusion. Germline filtered somatic mutational calls based on whole genome 642 sequencing were used for downstream analyses where individuals with fewer than 100 somatic 643 mutations were excluded (due to limitations in defining chromatin-state-specific mutational 644 effects). Each cancer type was treated as a single cohort, with the exception of breast cancer 645 (BRCA) where additional stratified analyses were performed according to major subgroups 646 (Luminal, ERBB2/Her2-positive, and triple negative breast cancers (TNBC)). The full list of 647 ICGC donor IDs and cohorts is included in Supplemental Table 1. A total of 2634 individuals 648 were included across all cancer types.

649

650 METABRIC expression, CNA, clinical, and survival data were downloaded from European 651 Genome-Phenome Archive (EGA). Data from The Cancer Genome Atlas were utilized for 
652 expression-survival validation (Liu et al., 2018) and CRISPR analyses (Bailey et al., 2018) and

653 PCAWG was used for CRISPR analyses (Rheinbay et al., 2020).

654

655 Defining chromatin state and open chromatin regions

656 Chromatin state annotations for all cancer types except prostate were downloaded from the

657 Roadmap Epigenomics Project integrated analyses while DNase hypersensitivity peaks for all

658 cancer types except prostate were downloaded from the ENCODE portal. For prostate cancer,

659 annotations were obtained from GEO:GSE63094 and quantized to chromatin states in 100bp

660 windows using ChromHMM, and used as annotation sources as described previously (Sallari et

661 al., 2017).

662

663 We used a stringent filtering step based on sequence uniqueness to avoid miscalling of

664 chromatin states. In brief, three filters were combined to eliminate regions that might have

665 artifactual annotations or missing genotype calls as a result of mappability bias. First, the

666 ENCODE blacklist regions and UCSC hg19 genome assembly gaps were merged together,

667 followed by looking in umap (ENCODE Project Consortium, 2012) and removing

668 non-uniquely-mappable regions. This results in approximately one third of the genome (mostly

669 centromeric and telomeric regions) being masked of repetitive regions.

671 Regional mutation rate estimation and null model mutation distribution

673 While replication timing data are available in some relevant cell types through ENCODE, the

674 vast majority of cancer types have no annotations available. As such, the regional mutation rate

675 was used as an estimate of replication timing, given their high correlation and reproducible

676 effects on mutational spectrum (Stamatoyannopoulos et al., 2009). Two distinct windows of

677 mutation counts were used -- $25 \mathrm{~kb}$ and $250 \mathrm{~kb}$-- and the counts were summed across patients

678 normalized by patient count (so that rates are comparable between cancer types), total number

679 of mutations in the patient, and the window size (to achieve comparable distributions for both

$68025 \mathrm{~kb}$ and $250 \mathrm{~kb}$ windows).

681

682 At every nucleotide in the genome, on a per-cancer-type basis, covariates were estimated as

683 the chromatin state (reduced to 7 states: promoter, enhancer, transcribed, repressed, bivalent,

684 heterochromatin, and quiescent), DNase hypersensitivity peaks, and estimated regional

685 mutation rate, the calculation of which is described above.

686

687 To ensure the robustness of results, all models were repeated with multiple regional mutation

688 rate windows and nucleotide fragment sizes. For the single nucleotide model, we ran models

689 corrected for stranded trinucleotide context (Alexandrov et al., 2013). Using these distributions,

690 we tested for the enrichment of mutations across active chromatin states. We focused on active

691 regulatory regions as these have previously been implicated in cancer development 
692 (Sabarinathan et al., 2016), and because epigenetic alterations in the cell of origin are thought

693 to potentiate cancer development via loss of tumor suppression (Garinis et al., 2002).

694

695 Mapping regulatory elements to genes

696 Regulatory elements were mapped to genes using Hi-C links, described above, as well as with 697 correlation-based links (Rheinbay et al., 2020) that utilize modules of co-activated enhancers 698 and co-expressed genes across the Roadmap RNA-seq profiled samples. In addition, the core 699 promoter region was added to the tests as relevant, using annotations from the FANTOM5

700 consortium (FANTOM Consortium and the RIKEN PMI and CLST (DGT) et al., 2014). Histone I 701 genes, immunoglobulin genes, HLA genes, non-coding "AC" genes, and RNA genes were

702 excluded from further analyses due to either their repetitive structure or lack of adequate

703 annotation coverage, respectively.

704

705 Promoter elements $(n=57,534)$ were defined based on the FANTOM5 consortium CAGE 706 sequencing (FANTOM Consortium and the RIKEN PMI and CLST (DGT) et al., 2014). Promoter 707 BED region defintions were then aggregated within each protein coding gene and intersected

708 with chromatin state annotations. Any elements overlapping with collapsed promoter/strong 709 enhancer (Tss or TssFInk) chromatin states were labeled as active promoters in downstream 710 analysis.

\section{Estimation of mutational overburdening}

713 Four tests were employed to estimate the overburdening of mutations. In the first approach, a

714 resampling strategy replaced each tile (a region of consecutive bases, between $1 \mathrm{bp}$ and 100bp)

715 in the aggregate regulatory landscape with one that has the same reference nucleotide context,

716 regional mutation rate, chromatin state, and open chromatin level. Then the number of

717 mutations is assessed and the significance is calculated through the empirical $p$-value relative to

718 the genomic background null distribution. This is exact and gives uninflated quantile-quantile

719 plots, but is computationally intensive to calculate, and thus all associations were first run using

720 the parametric models described below, and marginally significant associations were replicated

721 using the permutation test as a final filter. For evaluation of coding gene effects, q-values for

722 enrichment of putative cancer-mutated genes (Lawrence et al., 2014) were downloaded and

723 ordered by their pan-cancer q-value.

725 As a pre-filter for the pan-cancer runs, where non-parametric tests are prohibitively time

726 consuming, a poisson distribution is used, where the lambda parameter is estimated from the

727 genome-wide distribution of nucleotides that share the same covariates (regional mutation rate,

728 patient, chromatin state, and DNase sensitivity). Every nucleotide is assumed to be independent

729 and the product of the observed values is the overall expectation. 
731 In order to capture putative enriched genes which violate the poisson assumption, a z-score test

732 is used, where the mean mutation count was derived using the same covariates as the Poisson

733 test. Finally, the Cochran-Mantel-Haentzel $(\mathrm{CMH})$ test was used in which chromatin state strata

734 are simultaneously tested for having mutations at an odds ratio other than one. Together, these

735 three tests act as filters to identify only the gene-state-cancer type combinations most likely to

736 be enriched, and those combinations can be further refined using the non-parametric models.

738 For the non-parametric models, genomic windows of size $1 \mathrm{bp}, 10 \mathrm{bp}$, or $100 \mathrm{bp}$ were stratified by

739 canonical chromatin states and the presence of open chromatin, and within each, normalized

740 regional mutation rate (mutations per megabase per thousand donors) and reference

741 trinucleotide context were recorded. To evaluate a gene, the associated regulatory regions were

742 divided into chromatin states, and the number of tiles of a given size and parameters were

743 tallied. Then, for each permutation, random matched regions were regenerated and tallied from

744 covariate-matched regions of the same length across the genome and summed across the

745 regulatory landscape.

747 Fisher's method was used to combine p-values across cancer types. Under this model, we

748 assume that the estimates from the cancer types are independent given the lack of

749 individual-level overlap between studies of different cancer types.

\section{Bootstrap validation of mutation enrichment}

753 A validation of the mutation selection process was performed for the Breast cancer association

754 at IPO9. Individuals were resampled uniformly at random in the Basal breast cancer subtype

755 and the observed and expected number of mutations were recalculated. Resampling was

756 performed 20 times and the enrichment in both mutation counts (Supplemental Figure 2A) and

757 patient counts (Supplemental Figure 2B) were tallied.

759 Survival analyses

760 For the METABRIC cohort, clinical data, including relapse free survival was obtained from

761 (Rueda et al. Nature 2019), and expression and copy number from EGA. Expression of IPO9

762 was adjusted by copy number by regressing the copy number value from the expression.

763 Kaplan-Meier plots were generated with the package "survminer", where the top 1/3 and bottom

764 1/3 expression values for each gene were defined as high versus low, respectively. Cox

765 Proportional Hazards Models were generated using the CoxPH function in the survival package,

766 adjusting for relevant clinical covariates, including age, stage, grade, size, number of lymph

767 nodes positive, estrogen and progesterone receptor status, as well as HER2/ERBB2 status.

768 Estrogen receptor (ER) status was not included in the model for luminal tumors since most are

769 ER-positive. For the TCGA outcome analysis, clinical data (overall survival) was obtained from

770 (Liu et al. Cell 2018), and expression (FPKM, upper quantile) and copy number data from

771 gdc.cancer.gov. Expression was log2 transformed and scale normalized. Cox Proportional 
772 Hazards Models were generated similar to that for the METABRIC cohort, again adjusting for 773 clinical covariates (when available) including age, stage, gender and grade. Only tumor types

774 with sufficient numbers and follow-up times were used for the main analyses (Liu et al., 2018).

776 CRISPR screen and essentiality analyses

\section{References}

793 Alexandrov, L.B., Nik-Zainal, S., Wedge, D.C., Aparicio, S.A.J.R., Behjati, S., Biankin, A.V., 794 Bignell, G.R., Bolli, N., Borg, A., Børresen-Dale, A.-L., et al. (2013). Signatures of mutational 795 processes in human cancer. Nature 500, 415-421.

796 Araya, C.L., Cenik, C., Reuter, J.A., Kiss, G., Pande, V.S., Snyder, M.P., and Greenleaf, W.J. 797 (2016). Identification of significantly mutated regions across cancer types highlights a rich 798 landscape of functional molecular alterations. Nat. Genet. 48, 117-125.

799 Bailey, M.H., Tokheim, C., Porta-Pardo, E., Sengupta, S., Bertrand, D., Weerasinghe, A., 800 Colaprico, A., Wendl, M.C., Kim, J., Reardon, B., et al. (2018). Comprehensive Characterization 801 of Cancer Driver Genes and Mutations. Cell 173, 371-385.e18.

802 Bailey, S.D., Desai, K., Kron, K.J., Mazrooei, P., Sinnott-Armstrong, N.A., Treloar, A.E., Dowar, 803 M., Thu, K.L., Cescon, D.W., Silvester, J., et al. (2016). Noncoding somatic and inherited 804 single-nucleotide variants converge to promote ESR1 expression in breast cancer. Nat. Genet. 805 48, 1260-1266.

806 Berger, M.F., Hodis, E., Heffernan, T.P., Deribe, Y.L., Lawrence, M.S., Protopopov, A., Ivanova, 807 E., Watson, I.R., Nickerson, E., Ghosh, P., et al. (2012). Melanoma genome sequencing reveals 808 frequent PREX2 mutations. Nature 485, 502-506.

809 Boyle, E.A., Pritchard, J.K., and Greenleaf, W.J. (2018). High-resolution mapping of cancer cell 
810 networks using co-functional interactions.

811 Caridi, C.P., D’Agostino, C., Ryu, T., Zapotoczny, G., Delabaere, L., Li, X., Khodaverdian, V.Y., 812 Amaral, N., Lin, E., Rau, A.R., et al. (2018). Nuclear F-actin and myosins drive relocalization of 813 heterochromatic breaks. Nature 559, 54-60.

814 Chang, D.Y., Hsu, K., and Maraia, R.J. (1996). Monomeric scAlu and nascent dimeric Alu RNAs 815 induced by adenovirus are assembled into SRP9/14-containing RNPs in HeLa cells. Nucleic 816 Acids Res. 24, 4165-4170.

817 Cho, S.W., Xu, J., Sun, R., Mumbach, M.R., Carter, A.C., Chen, Y.G., Yost, K.E., Kim, J., He, J., 818 Nevins, S.A., et al. (2018). Promoter of IncRNA Gene PVT1 Is a Tumor-Suppressor DNA

819 Boundary Element. Cell 173, 1398-1412.e22.

820 Curtis, C., Shah, S.P., Chin, S.-F., Turashvili, G., Rueda, O.M., Dunning, M.J., Speed, D., Lynch, 821 A.G., Samarajiwa, S., Yuan, Y., et al. (2012). The genomic and transcriptomic architecture of 8222,000 breast tumours reveals novel subgroups. Nature 486, 346-352.

823 David, C.J., and Manley, J.L. (2010). Alternative pre-mRNA splicing regulation in cancer: 824 pathways and programs unhinged. Genes Dev. 24, 2343-2364.

825 Degner, J.F., Pai, A.A., Pique-Regi, R., Veyrieras, J.-B., Gaffney, D.J., Pickrell, J.K., De Leon, 826 S., Michelini, K., Lewellen, N., Crawford, G.E., et al. (2012). DNase I sensitivity QTLs are a 827 major determinant of human expression variation. Nature 482, 390-394.

828 De Ingeniis, J., Ratnikov, B., Richardson, A.D., Scott, D.A., Aza-Blanc, P., De, S.K., Kazanov, 829 M., Pellecchia, M., Ronai, Z. 'ev, Osterman, A.L., et al. (2012). Functional specialization in 830 proline biosynthesis of melanoma. PLoS One 7, e45190.

831 Denisova, E., Heidenreich, B., Nagore, E., Rachakonda, P.S., Hosen, I., Akrap, I., Traves, V., 832 García-Casado, Z., López-Guerrero, J.A., Requena, C., et al. (2015). Frequent DPH3 promoter 833 mutations in skin cancers. Oncotarget 6, 35922-35930.

834 Dopie, J., Skarp, K.-P., Rajakylä, E.K., Tanhuanpää, K., and Vartiainen, M.K. (2012). Active 835 maintenance of nuclear actin by importin 9 supports transcription. Proc. Natl. Acad. Sci. U. S. A. 836 109, E544-E552.

837 Elliott, L.T., Sharp, K., Alfaro-Almagro, F., Shi, S., Miller, K.L., Douaud, G., Marchini, J., and 838 Smith, S.M. (2018). Genome-wide association studies of brain imaging phenotypes in UK 839 Biobank. Nature 562, 210-216.

840 ENCODE Project Consortium (2012). An integrated encyclopedia of DNA elements in the 841 human genome. Nature 489, 57-74.

842 FANTOM Consortium and the RIKEN PMI and CLST (DGT), Forrest, A.R.R., Kawaji, H., Rehli, 843 M., Baillie, J.K., de Hoon, M.J.L., Haberle, V., Lassmann, T., Kulakovskiy, I.V., Lizio, M., et al. 844 (2014). A promoter-level mammalian expression atlas. Nature 507, 462-470.

845 Feigin, M.E., Garvin, T., Bailey, P., Waddell, N., Chang, D.K., Kelley, D.R., Shuai, S., Gallinger, 846 S., McPherson, J.D., Grimmond, S.M., et al. (2017). Recurrent noncoding regulatory mutations 
847 in pancreatic ductal adenocarcinoma. Nat. Genet. 49, 825-833.

848 Ferrari, A., Vincent-Salomon, A., Pivot, X., Sertier, A.-S., Thomas, E., Tonon, L., Boyault, S.,

849 Mulugeta, E., Treilleux, I., MacGrogan, G., et al. (2016). A whole-genome sequence and

850 transcriptome perspective on HER2-positive breast cancers. Nat. Commun. 7, 12222.

851 Fishilevich, S., Nudel, R., Rappaport, N., Hadar, R., Plaschkes, I., Iny Stein, T., Rosen, N.,

852 Kohn, A., Twik, M., Safran, M., et al. (2017). GeneHancer: genome-wide integration of

853 enhancers and target genes in GeneCards. Database 2017.

854 Flavahan, W.A., Drier, Y., Liau, B.B., Gillespie, S.M., Venteicher, A.S., Stemmer-Rachamimov, 855 A.O., Suvà, M.L., and Bernstein, B.E. (2016). Insulator dysfunction and oncogene activation in 856 IDH mutant gliomas. Nature 529, 110-114.

857 Forbes, S.A., Beare, D., Boutselakis, H., Bamford, S., Bindal, N., Tate, J., Cole, C.G., Ward, S., 858 Dawson, E., Ponting, L., et al. (2017). COSMIC: somatic cancer genetics at high-resolution.

859 Nucleic Acids Res. 45, D777-D783.

860 Gao, J., Aksoy, B.A., Dogrusoz, U., Dresdner, G., Gross, B., Sumer, S.O., Sun, Y., Jacobsen, 861 A., Sinha, R., Larsson, E., et al. (2013). Integrative analysis of complex cancer genomics and 862 clinical profiles using the cBioPortal. Sci. Signal. 6, I1.

863 Garinis, G.A., Patrinos, G.P., Spanakis, N.E., and Menounos, P.G. (2002). DNA

864 hypermethylation: when tumour suppressor genes go silent. Hum. Genet. 111, 115-127.

865 Gasperini, M., Hill, A.J., McFaline-Figueroa, J.L., Martin, B., Kim, S., Zhang, M.D., Jackson, D., 866 Leith, A., Schreiber, J., Noble, W.S., et al. (2019). A Genome-wide Framework for Mapping

867 Gene Regulation via Cellular Genetic Screens. Cell 176, 377-390.e19.

868 Guo, Y., Updegraff, B.L., Park, S., Durakoglugil, D., Cruz, V.H., Maddux, S., Hwang, T.H., and

869 O'Donnell, K.A. (2016). Comprehensive Ex Vivo Transposon Mutagenesis Identifies Genes That

870 Promote Growth Factor Independence and Leukemogenesis. Cancer Res. 76, 773-786.

871 Hanahan, D., and Weinberg, R.A. (2011). Hallmarks of cancer: the next generation. Cell 144, $872646-674$.

873 Hnisz, D., Weintraub, A.S., Day, D.S., Valton, A.-L., Bak, R.O., Li, C.H., Goldmann, J., Lajoie, 874 B.R., Fan, Z.P., Sigova, A.A., et al. (2016). Activation of proto-oncogenes by disruption of 875 chromosome neighborhoods. Science 351, 1454-1458.

876 Horn, S., Figl, A., Rachakonda, P.S., Fischer, C., Sucker, A., Gast, A., Kadel, S., Moll, I., 877 Nagore, E., Hemminki, K., et al. (2013). TERT promoter mutations in familial and sporadic 878 melanoma. Science 339, 959-961.

879 Huang, F.W., Hodis, E., Xu, M.J., Kryukov, G.V., Chin, L., and Garraway, L.A. (2013). Highly 880 recurrent TERT promoter mutations in human melanoma. Science 339, 957-959.

881 ICGC/TCGA Pan-Cancer Analysis of Whole Genomes Consortium (2020). Pan-cancer analysis 882 of whole genomes. Nature 578, 82-93.

883 Jo, U., Cai, W., Wang, J., Kwon, Y., D’Andrea, A.D., and Kim, H. (2016). PCNA-Dependent 
884 Cleavage and Degradation of SDE2 Regulates Response to Replication Stress. PLoS Genet.

88512 , e1006465.

886 Joshi, A.A., and Struhl, K. (2005). Eaf3 chromodomain interaction with methylated H3-K36 links

887 histone deacetylation to Pol II elongation. Mol. Cell 20, 971-978.

888 Kasowski, M., Kyriazopoulou-Panagiotopoulou, S., Grubert, F., Zaugg, J.B., Kundaje, A., Liu, Y., 889 Boyle, A.P., Zhang, Q.C., Zakharia, F., Spacek, D.V., et al. (2013). Extensive variation in 890 chromatin states across humans. Science 342, 750-752.

891 Kilpinen, H., Waszak, S.M., Gschwind, A.R., Raghav, S.K., Witwicki, R.M., Orioli, A., 892 Migliavacca, E., Wiederkehr, M., Gutierrez-Arcelus, M., Panousis, N.I., et al. (2013).

893 Coordinated effects of sequence variation on DNA binding, chromatin structure, and 894 transcription. Science 342, 744-747.

895 Koedoot, E., Wolters, L., van de Water, B., and Dévédec, S.E.L. (2019). Splicing regulatory 896 factors in breast cancer hallmarks and disease progression. Oncotarget 10, 6021-6037.

897 Lawrence, M.S., Stojanov, P., Mermel, C.H., Robinson, J.T., Garraway, L.A., Golub, T.R., 898 Meyerson, M., Gabriel, S.B., Lander, E.S., and Getz, G. (2014). Discovery and saturation 899 analysis of cancer genes across 21 tumour types. Nature 505, 495-501.

900 Leiserson, M.D.M., Blokh, D., Sharan, R., and Raphael, B.J. (2013). Simultaneous identification 901 of multiple driver pathways in cancer. PLoS Comput. Biol. 9, e1003054.

902 Lek, M., Karczewski, K.J., Minikel, E.V., Samocha, K.E., Banks, E., Fennell, T., O’Donnell-Luria, 903 A.H., Ware, J.S., Hill, A.J., Cummings, B.B., et al. (2016). Analysis of protein-coding genetic 904 variation in 60,706 humans. Nature 536, 285-291.

905 Liu, J., Lichtenberg, T., Hoadley, K.A., Poisson, L.M., Lazar, A.J., Cherniack, A.D., Kovatich, 906 A.J., Benz, C.C., Levine, D.A., Lee, A.V., et al. (2018). An Integrated TCGA Pan-Cancer Clinical 907 Data Resource to Drive High-Quality Survival Outcome Analytics. Cell 173, 400-416.e11.

908 Lobry, C., Oh, P., and Aifantis, I. (2011). Oncogenic and tumor suppressor functions of Notch in 909 cancer: it's NOTCH what you think. J. Exp. Med. 208, 1931-1935.

910 Lowdon, R.F., and Wang, T. (2017). Epigenomic annotation of noncoding mutations identifies 911 mutated pathways in primary liver cancer. PLoS One 12, e0174032.

912 Matsumiya, T., Xing, F., Ebina, M., Hayakari, R., Imaizumi, T., Yoshida, H., Kikuchi, H., Topham, 913 M.K., Satoh, K., and Stafforini, D.M. (2013). Novel role for molecular transporter importin 9 in 914 posttranscriptional regulation of IFN- $\varepsilon$ expression. J. Immunol. 191, 1907-1915.

915 Maurano, M.T., Humbert, R., Rynes, E., Thurman, R.E., Haugen, E., Wang, H., Reynolds, A.P., 916 Sandstrom, R., Qu, H., Brody, J., et al. (2012). Systematic localization of common 917 disease-associated variation in regulatory DNA. Science 337, 1190-1195.

918 McVicker, G., van de Geijn, B., Degner, J.F., Cain, C.E., Banovich, N.E., Raj, A., Lewellen, N., 919 Myrthil, M., Gilad, Y., and Pritchard, J.K. (2013). Identification of genetic variants that affect 920 histone modifications in human cells. Science 342, 747-749. 
931 Nik-Zainal, S., Davies, H., Staaf, J., Ramakrishna, M., Glodzik, D., Zou, X., Martincorena, I., 932 Alexandrov, L.B., Martin, S., Wedge, D.C., et al. (2016). Landscape of somatic mutations in 560 933 breast cancer whole-genome sequences. Nature 534, 47-54.

934 Osborne, N.J., Gurrin, L.C., Allen, K.J., Constantine, C.C., Delatycki, M.B., McLaren, C.E., 935 Gertig, D.M., Anderson, G.J., Southey, M.C., Olynyk, J.K., et al. (2010). HFE C282Y

936 homozygotes are at increased risk of breast and colorectal cancer. Hepatology 51, 1311-1318.

937 Pearson, J.L., Robinson, T.J., Muñoz, M.J., Kornblihtt, A.R., and Garcia-Blanco, M.A. (2008).

938 Identification of the cellular targets of the transcription factor TCERG1 reveals a prevalent role in 939 mRNA processing. J. Biol. Chem. 283, 7949-7961.

940 Rao, S.S.P., Huntley, M.H., Durand, N.C., Stamenova, E.K., Bochkov, I.D., Robinson, J.T., 941 Sanborn, A.L., Machol, I., Omer, A.D., Lander, E.S., et al. (2014). A 3D map of the human 942 genome at kilobase resolution reveals principles of chromatin looping. Cell 159, 1665-1680.

943 Rheinbay, E., Parasuraman, P., Grimsby, J., Tiao, G., Engreitz, J.M., Kim, J., Lawrence, M.S., 944 Taylor-Weiner, A., Rodriguez-Cuevas, S., Rosenberg, M., et al. (2017). Recurrent and functional 945 regulatory mutations in breast cancer. Nature 547, 55-60.

946 Rheinbay, E., Nielsen, M.M., Abascal, F., Wala, J.A., Shapira, O., Tiao, G., Hornshøj, H., Hess, 947 J.M., Juul, R.I., Lin, Z., et al. (2020). Analyses of non-coding somatic drivers in 2,658 cancer 948 whole genomes. Nature 578, 102-111.

949 Roadmap Epigenomics Consortium, Kundaje, A., Meuleman, W., Ernst, J., Bilenky, M., Yen, A., 950 Heravi-Moussavi, A., Kheradpour, P., Zhang, Z., Wang, J., et al. (2015). Integrative analysis of 951111 reference human epigenomes. Nature 518, 317-330.

952 Rueda, O.M., Sammut, S.-J., Seoane, J.A., Chin, S.-F., Caswell-Jin, J.L., Callari, M., Batra, R., 953 Pereira, B., Bruna, A., Ali, H.R., et al. (2019). Dynamics of breast-cancer relapse reveal 954 late-recurring ER-positive genomic subgroups. Nature 567, 399-404.

955 Sabarinathan, R., Mularoni, L., Deu-Pons, J., Gonzalez-Perez, A., and López-Bigas, N. (2016). 956 Nucleotide excision repair is impaired by binding of transcription factors to DNA. Nature 532, 957 264-267.

958 Sack, L.M., Davoli, T., Li, M.Z., Li, Y., Xu, Q., Naxerova, K., Wooten, E.C., Bernardi, R.J., Martin, 
959 T.D., Chen, T., et al. (2018). Profound Tissue Specificity in Proliferation Control Underlies

960 Cancer Drivers and Aneuploidy Patterns. Cell 173, 499-514.e23.

961 Sallari, R.C., Sinnott-Armstrong, N.A., French, J.D., Kron, K.J., Ho, J., Moore, J.H., Stambolic,

962 V., Edwards, S.L., Lupien, M., and Kellis, M. (2017). Convergence of dispersed regulatory

963 mutations predicts driver genes in prostate cancer.

964 Schaub, C., Nagaso, H., Jin, H., and Frasch, M. (2012). Org-1, the Drosophila ortholog of Tbx1, 965 is a direct activator of known identity genes during muscle specification. Development 139,

966 1001-1012.

967 Schmidt, D., Wilson, M.D., Ballester, B., Schwalie, P.C., Brown, G.D., Marshall, A., Kutter, C.,

968 Watt, S., Martinez-Jimenez, C.P., Mackay, S., et al. (2010). Five-vertebrate ChIP-seq reveals

969 the evolutionary dynamics of transcription factor binding. Science 328, 1036-1040.

970 Sharili, A.S., Kenny, F.N., Vartiainen, M.K., and Connelly, J.T. (2016). Nuclear actin modulates

971 cell motility via transcriptional regulation of adhesive and cytoskeletal genes. Sci. Rep. 6, 33893.

972 Stamatoyannopoulos, J.A., Adzhubei, I., Thurman, R.E., Kryukov, G.V., Mirkin, S.M., and

973 Sunyaev, S.R. (2009). Human mutation rate associated with DNA replication timing. Nat. Genet.

974 41, 393-395.

975 Takeda, D.Y., Spisák, S., Seo, J.-H., Bell, C., O’Connor, E., Korthauer, K., Ribli, D., Csabai, I.,

976 Solymosi, N., Szállási, Z., et al. (2018). A Somatically Acquired Enhancer of the Androgen

977 Receptor Is a Noncoding Driver in Advanced Prostate Cancer. Cell 174, 422-432.e13.

978 Tong, P., Monahan, J., and Prendergast, J.G.D. (2017). Shared regulatory sites are abundant in

979 the human genome and shed light on genome evolution and disease pleiotropy. PLoS Genet.

980 13, e1006673.

981 Viita, T., Kyheröinen, S., Prajapati, B., Virtanen, J., Frilander, M.J., Varjosalo, M., and Vartiainen, 982 M.K. (2019). Nuclear actin interactome analysis links actin to KAT14 histone acetyl transferase 983 and mRNA splicing. J. Cell Sci. 132.

984 Vinagre, J., Almeida, A., Pópulo, H., Batista, R., Lyra, J., Pinto, V., Coelho, R., Celestino, R., 985 Prazeres, H., Lima, L., et al. (2013). Frequency of TERT promoter mutations in human cancers.

986 Nat. Commun. 4, 2185.

987 Wagle, N., Painter, C., Krevalin, M., Oh, C., Anderka, K., Larkin, K., Lennon, N., Dillon, D.,

988 Frank, E., Winer, E.P., et al. (2016). The Metastatic Breast Cancer Project: A national

989 direct-to-patient initiative to accelerate genomics research. J. Clin. Orthod. 34,

990 LBA1519-LBA1519.

991 Watson, I.R., Takahashi, K., Futreal, P.A., and Chin, L. (2013). Emerging patterns of somatic 992 mutations in cancer. Nat. Rev. Genet. 14, 703-718.

993 Weinhold, N., Jacobsen, A., Schultz, N., Sander, C., and Lee, W. (2014). Genome-wide analysis 994 of noncoding regulatory mutations in cancer. Nat. Genet. 46, 1160-1165.

995 Wu, K.J., Grandori, C., Amacker, M., Simon-Vermot, N., Polack, A., Lingner, J., and 
996 Dalla-Favera, R. (1999). Direct activation of TERT transcription by c-MYC. Nat. Genet. 21,

$997220-224$.

998 Zehir, A., Benayed, R., Shah, R.H., Syed, A., Middha, S., Kim, H.R., Srinivasan, P., Gao, J.,

999 Chakravarty, D., Devlin, S.M., et al. (2017). Mutational landscape of metastatic cancer revealed

1000 from prospective clinical sequencing of 10,000 patients. Nat. Med. 23, 703-713.

1001 Zhang, W., Bojorquez-Gomez, A., Velez, D.O., Xu, G., Sanchez, K.S., Shen, J.P., Chen, K., 1002 Licon, K., Melton, C., Olson, K.M., et al. (2018). A global transcriptional network connecting 1003 noncoding mutations to changes in tumor gene expression. Nat. Genet. 50, 613-620.

1004 Zhou, S., Hawley, J.R., Soares, F., Grillo, G., Teng, M., Madani Tonekaboni, S.A., Hua, J.T., 1005 Kron, K.J., Mazrooei, P., Ahmed, M., et al. (2020). Noncoding mutations target cis-regulatory 1006 elements of the FOXA1 plexus in prostate cancer. Nat. Commun. 11, 441.

1007 Zhou, X., Li, D., Zhang, B., Lowdon, R.F., Rockweiler, N.B., Sears, R.L., Madden, P.A.F., 1008 Smirnov, I., Costello, J.F., and Wang, T. (2015). Epigenomic annotation of genetic variants using 1009 the Roadmap Epigenome Browser. Nat. Biotechnol. 33, 345-346.

1010 Zhu, H., Uusküla-Reimand, L., Isaev, K., Wadi, L., Alizada, A., Shuai, S., Huang, V.,

1011 Aduluso-Nwaobasi, D., Paczkowska, M., Abd-Rabbo, D., et al. (2020). Candidate Cancer Driver

1012 Mutations in Distal Regulatory Elements and Long-Range Chromatin Interaction Networks. Mol.

1013 Cell. 


\section{Figure 1: Model for aggregating mutations in gene-associated regulatory regions.}

A. Study overview

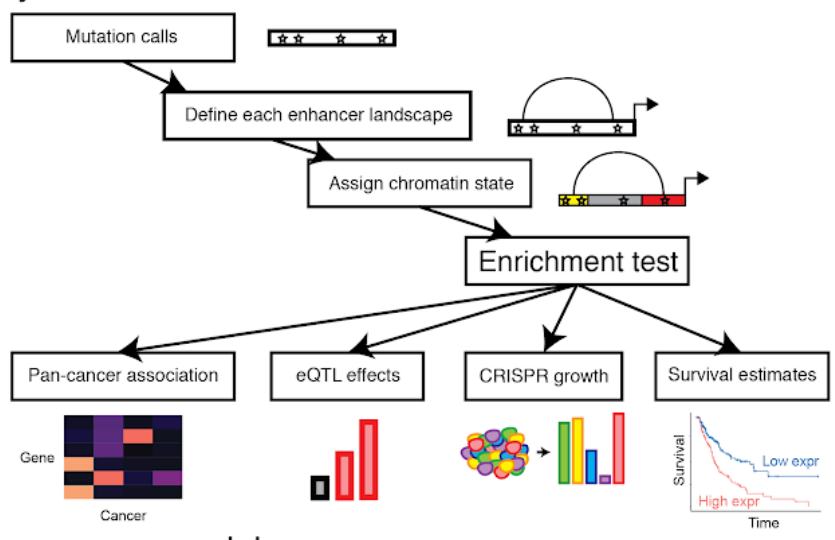

B. Convergence model

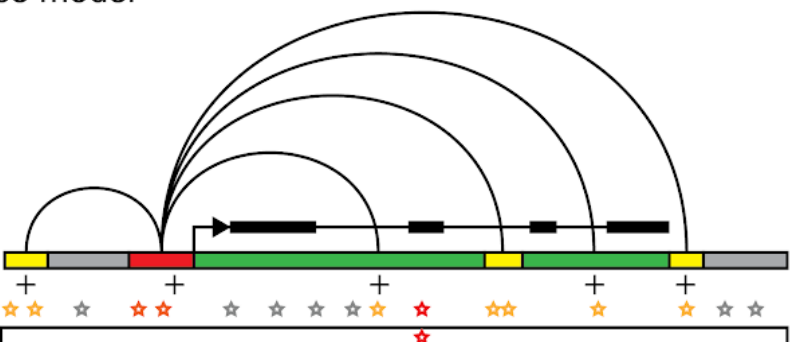
All mutations:

Regulatory mutations: Background mutations:

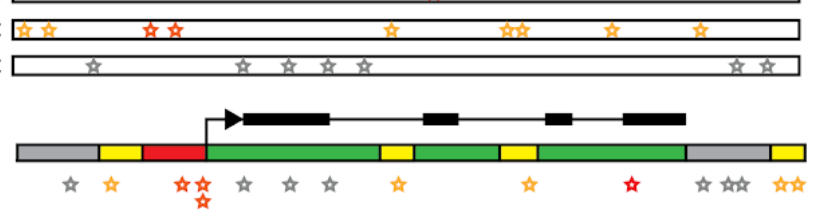

C. Regulatory mutation distribution
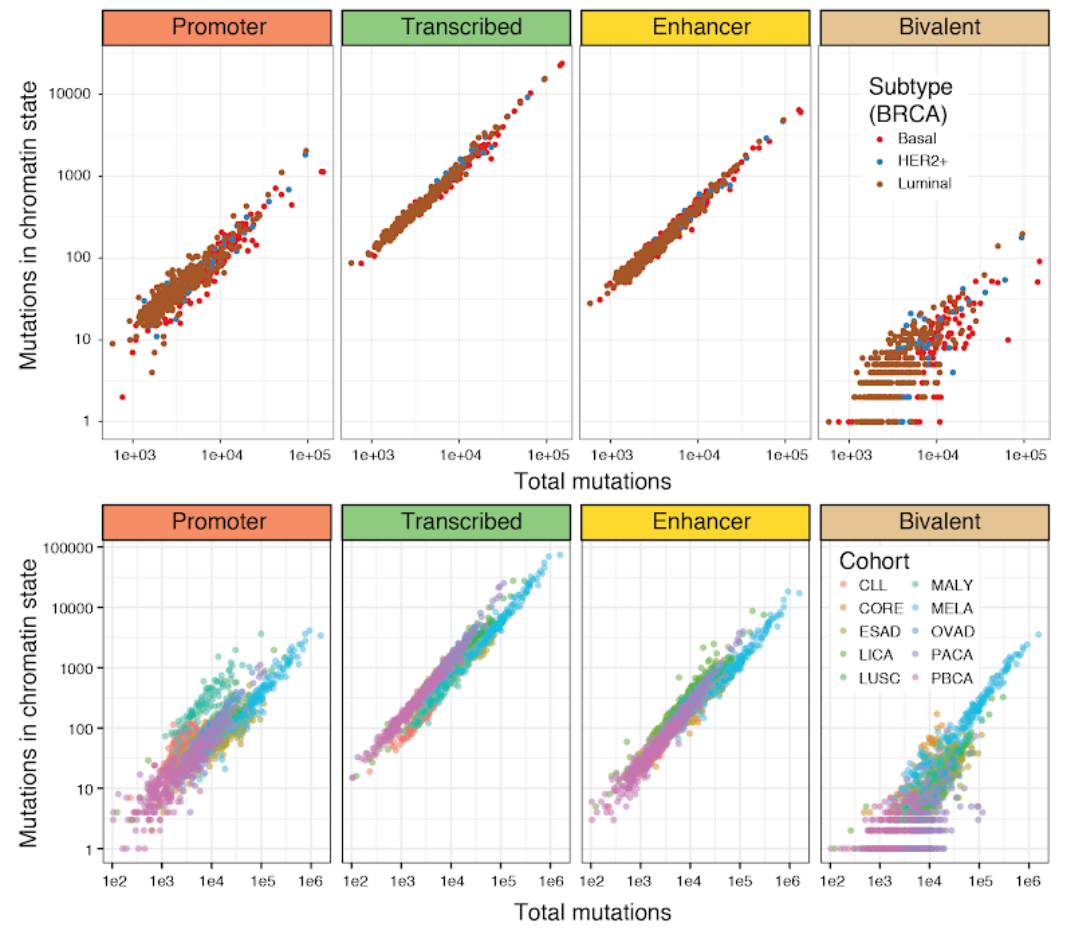
1017

1018

1019

1020

1021

1022

1023

1024

1025

1026

1027

1028

1029

1030

1031

1032

A. Study overview. Overview of approach to evaluate aggregate mutational burden in non-coding regulatory regions across cancer types, their functional effects, and clinical outcome associations.

B. Convergence model. Mutations accumulate in coding sequences and promoters, as detected in existing methods, but non-promoter regulatory mutations are likely spread across enhancer elements. Jointly testing specific regulatory regions can therefore increase the signal of mutational burden at a given gene, similar to an exome burden test. Both mutations and regulatory annotations change between tumor types.

C. Regulatory mutation distribution. Ordered distribution of mutation counts per individual for each of the cancer types studied in active and bivalent chromatin state annotations. $(x)$ axis total mutations for a given tumor, and $(y)$ axis number of mutations in a given chromatin state (promoter, enhancer, transcribed, or bivalent) for this tumor. Each point represents a single tumor within each subplot. For breast cancer, the three subtypes analysed separately are individually plotted. 


\section{Figure 2: Recurrent regulatory mutations in breast cancer.}

A. Breast cancer promoter enriched genes

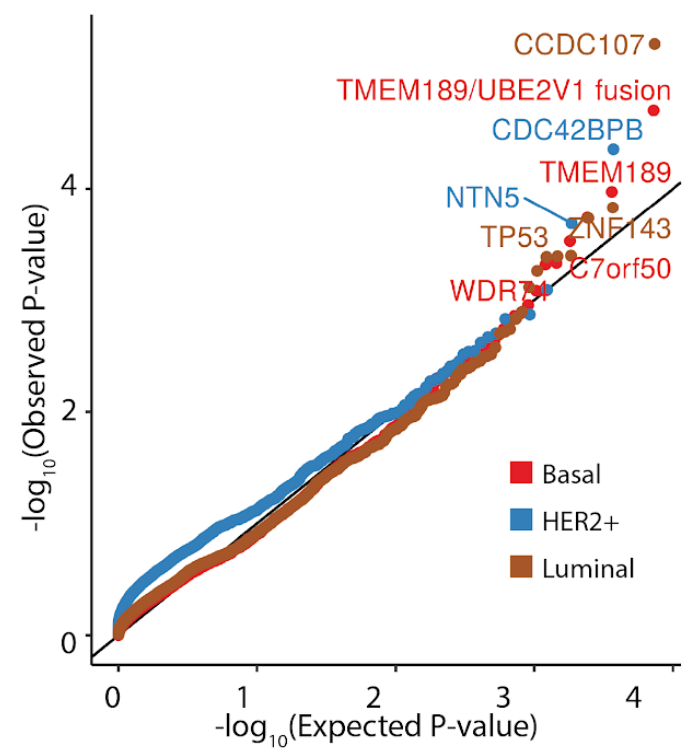

B. Breast cancer distal element enriched genes

\section{4}

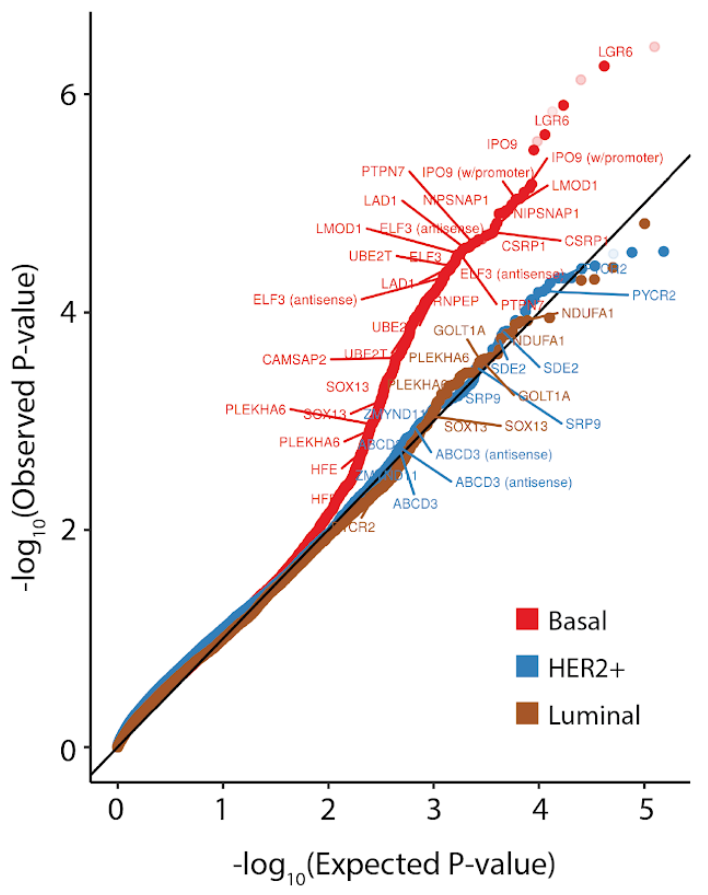

C. Subgroup combined distal element enriched genes

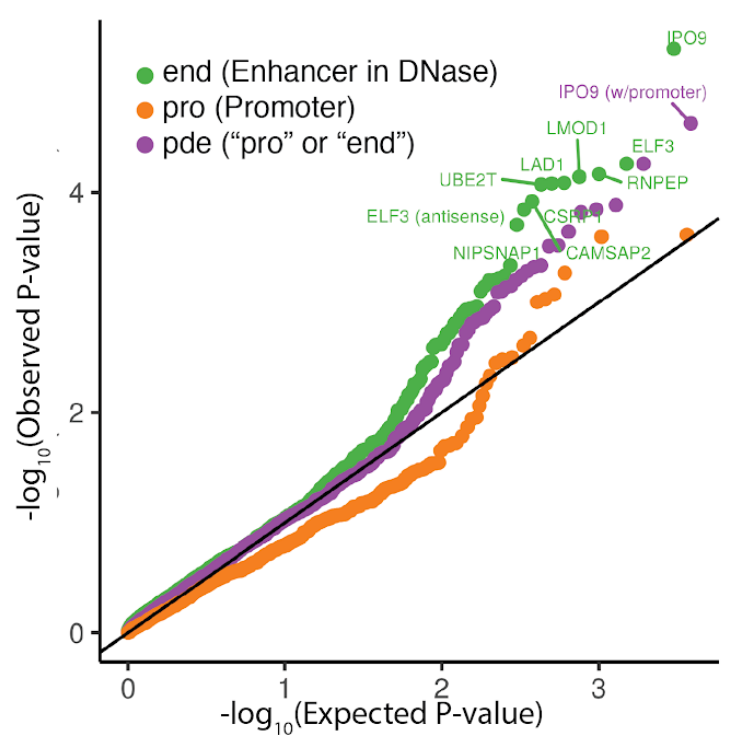

D. Enrichment of mutations across subtypes

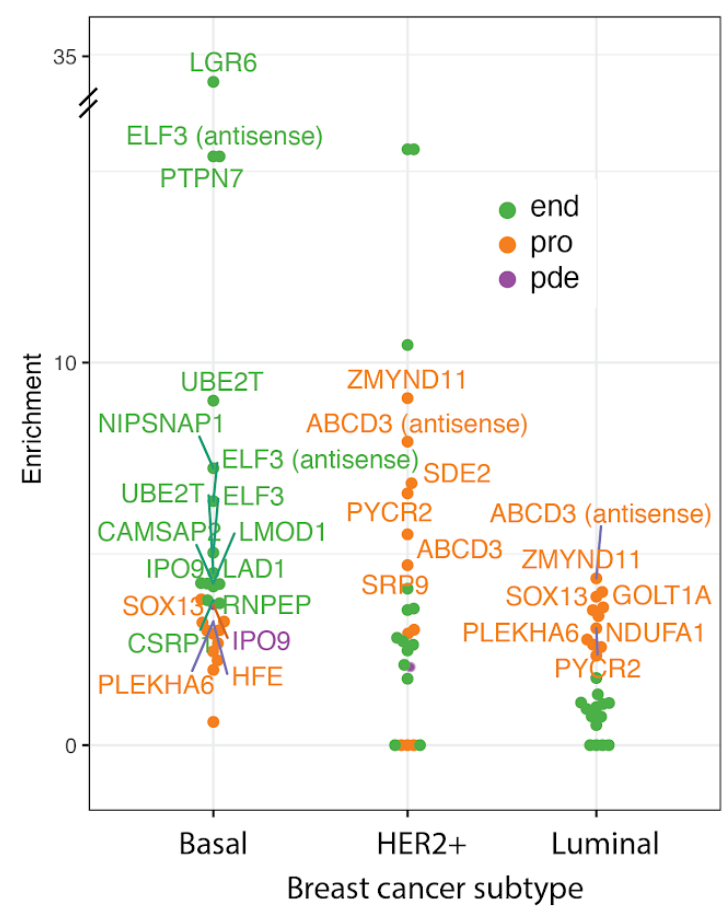

1035

1036

1037

1038

1039

1040

1041

1042

A. Breast cancer promoter enriched genes. Quantile-quantile plots of promoter mutations across breast subtypes (Basal, Luminal, HER2+).

B. Breast cancer distal element enriched genes. Quantile-quantile plots of distal regulatory mutations in each breast cancer subtype.

C. Subgroup combined distal element enriched genes. Quantile-quantile plot of different regulatory states, combined across subtypes. Only element-level definitions are shown, either enhancer and DNase (end), promoter or enhancer in DNase (pde), or promoter regardless of DNase status (pro). 
D. Enrichment of mutations across subtypes. Enrichment of significantly associated states from the combined analysis. Each dot within a given cancer type represents a single significantly associated gene, and each gene is repeated across all three cohorts to show relative enrichments of associated genes. Only element-level definitions are shown, either enhancer and DNase (end), promoter or DNase enhancer (pde), or promoter regardless of DNase (pro). Note that the promoter chromatin state is frequently observed in highly active enhancer elements as well as promoters themselves. 
bioRxiv preprint doi: https://doi.org/10.1101/2020.08.21.239954; this version posted August 31, 2020. The copyright holder for this preprint (which was not certified by peer review) is the author/funder, who has granted bioRxiv a license to display the preprint in perpetuity. It is made available under aCC-BY-NC-ND 4.0 International license.

\section{Figure 3: IPO9 is recurrently altered in breast cancer.}

A. IPO9 is amplified in breast cancer

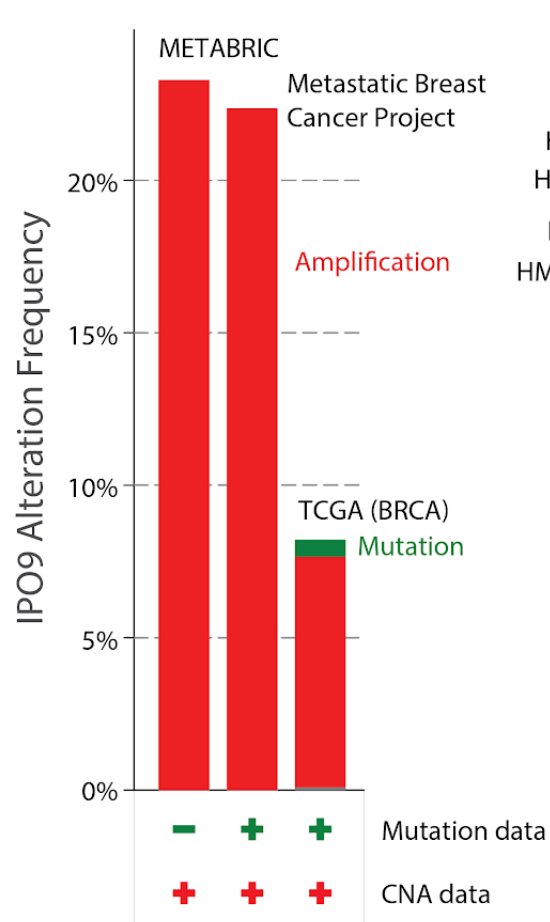

C. Epigenetic context at the IPO9 locus

chr1

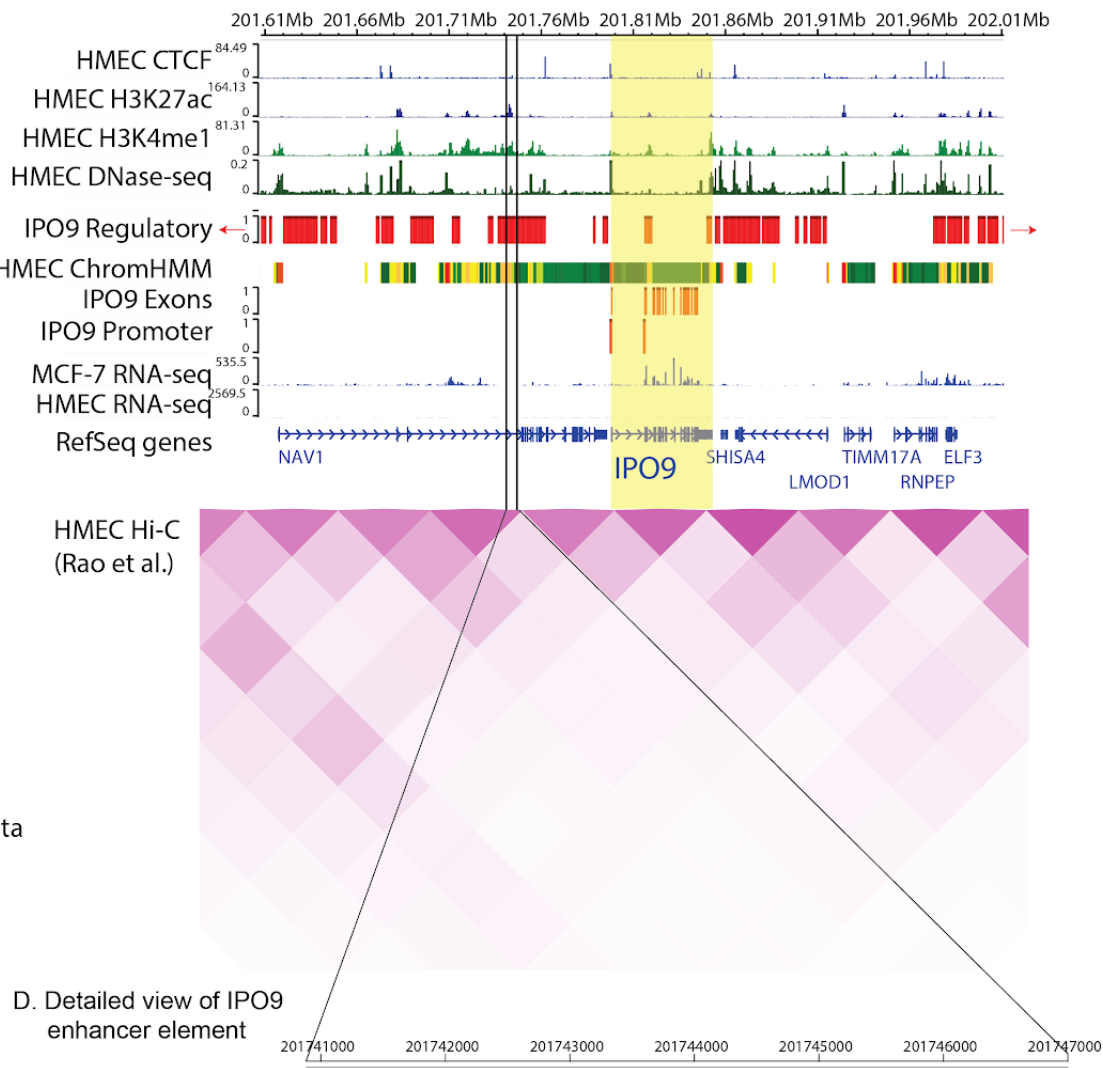

B. Aggregate regulatory mutated donors in replication cohorts

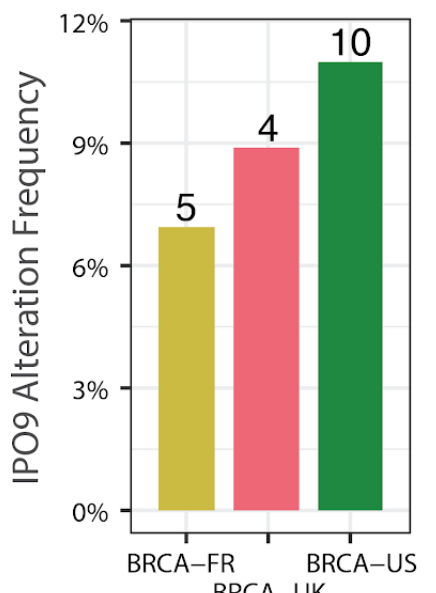

D. Detailed view of IPO9 enhancer element

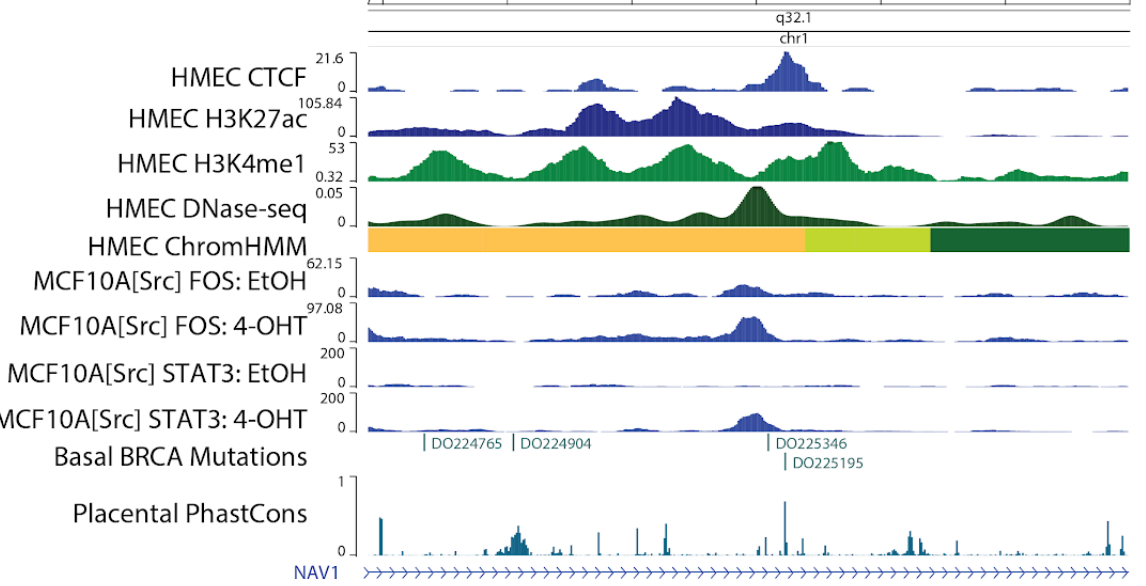

A. IPO9 is amplified in breast cancer. IPO9 is frequently amplified in breast cancer across three non-overlapping cohorts: METABRIC (Curtis et al., 2012; Rueda et al., 2019), the Metastatic Breast Cancer Project (Wagle et al., 2016), and The Cancer Genome Atlas (Gao et al., 2013; Liu et al., 2018). There are very few coding mutations in the Metastatic Breast Cancer Project and TCGA.

B. Aggregate regulatory mutate donors in replication cohorts. IPO9 regulatory region mutations were evaluated in three whole-genome sequenced validation cohorts: BRCA-UK and BRCA-US from PCAWG (ICGC/TCGA Pan-Cancer Analysis of Whole Genomes Consortium, 2020), and BRCA-FR (HER2+ amplified donors) from ICGC 
(Ferrari et al., 2016). Y axis, fraction of donors with regulatory mutations, with number of mutated donors shown above each bar. All three cohorts show a consistent proportion of donors ( 9\%) with mutations in DNase-hypersensitive, enhancer marked regions associated with the IPO9 promoter.

C. Epigenetic context at the IPO9 locus. ChIP-seq of histone modifications and CTCF, and open chromatin measured with DNase-seq, in human mammary epithelial cells (HMECs) are shown, as well as the aggregated chromatin state annotations in the two HMEC samples from Roadmap. In addition, the coding and non-coding elements tested for IPO9 are also indicated in red, and the expression of genes in the region is shown for both HMEC cells and MCF-7 breast cancer cells, showing the striking increased expression in MCF-7. Hi-C of HMEC cells (Rao et al., 2014) reveals a domain spanning the majority of regulatory elements (Zhou et al., 2015).

D. Detailed view of IPO9 enhancer elements. Detailed view of mutational context at an active element in an intron of NAV1. The 4-OHT response ChIP-seq profiles in MCF-7 cells and conservation tracks indicates that mutations are primarily located in regions of high activity or conservation. 
1081 Figure 4: Pan-cancer regulatory mutations have downstream effects on gene expression.

A. Pan-cancer putative regulatory driver genes

1082

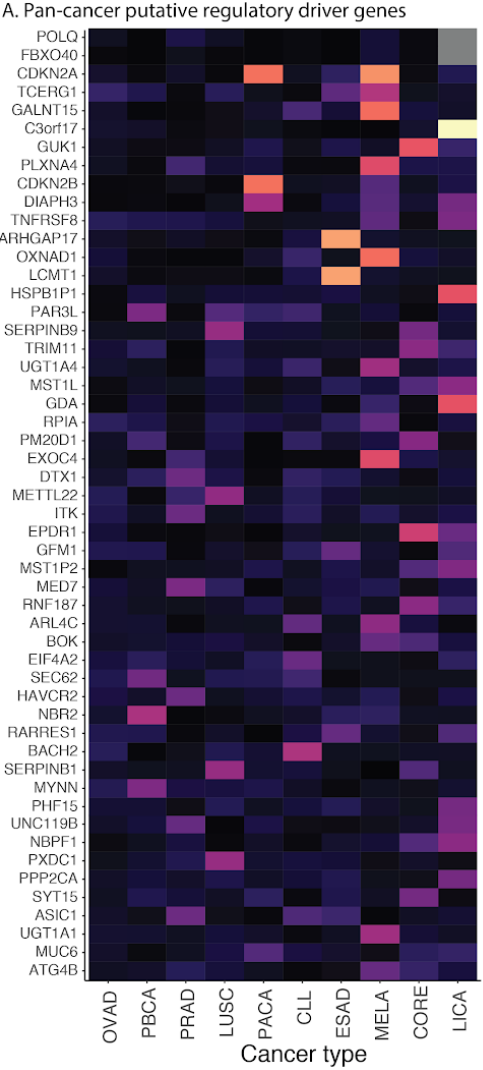

B. Multiple cancer associations by stringency

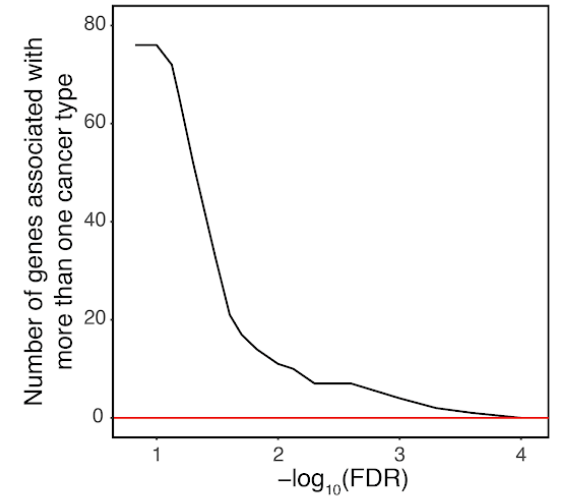

D. Lens-and-scallop plot of NBR2 mutations

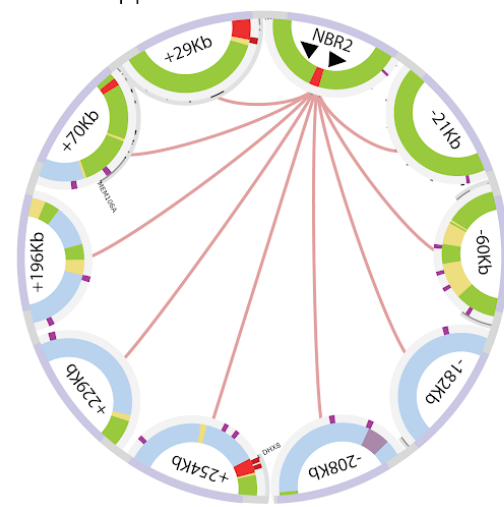

C. Downstream expression OTLs of mutations

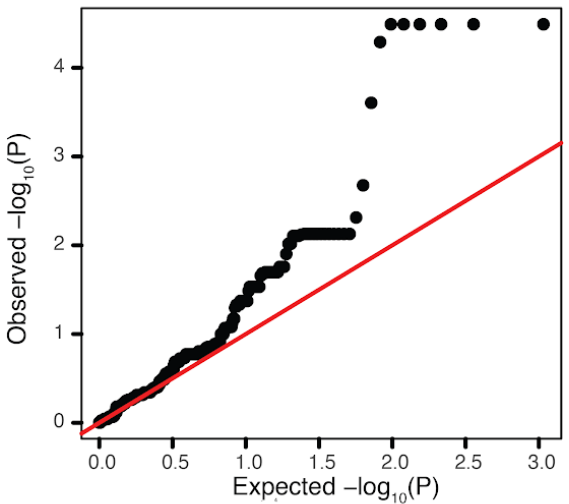

E. Lens-and-scallop plot of $\mathrm{CDH} 13$ mutations

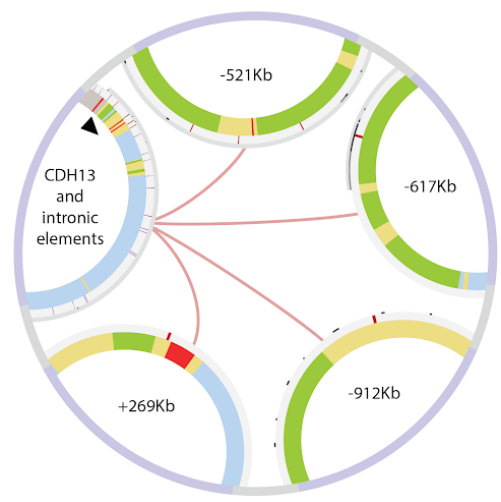

1083

1084

1085

1086

1087

1088

1089

1090

1091

1092

1093

1094

1095

1096

1097

1098

1099

1100

1101

1102

A. Pan-cancer putative regulatory driver genes. The shared landscape of regulatory alterations. Individual cancer types exhibit some uniquely significant genes, whereas other genes are recurrently mutated across cancer types.

B. Multiple cancer associations by stringency. Recurrence of genes across cancer types. Even at increasingly stringent FDR cutoffs, many genes harbor recurrent aggregated regulatory mutations across multiple cancer types.

C. Expression QTLs for recurrently mutated regulatory regions. Overall association of recurrently mutated genes with expression changes. The quantile-quantile plot shows significant changes in expression, as inferred from RNA-seq expression data of mutated versus non-mutated individuals.

D. Pan-cancer lens-and-scallop plot of NBR2 mutations. Variants are marked with red lines on the outer circle, with regions around mutated regulatory elements shown. Inner circles depict the chromatin state annotations corresponding to the mutated elements. Innermost black arrows at the gene locus mark promoters of BRCA1 and NBR2.

E. Pan-cancer lens-and-scallop plot of $\mathrm{CDH13}$ mutations. Variants are marked with red lines on the outer circle, with regions around mutated regulatory elements shown. Inner circles depict the chromatin state annotations corresponding to the mutated elements. Intronic elements are shown on gene locus for brevity. Innermost black arrow on gene locus marks promoter of $C D H 13$. 
bioRxiv preprint doi: https://doi.org/10.1101/2020.08.21.239954; this version posted August 31,2020. The copyright holder for this preprint (which was not certified by peer review) is the author/funder, who has granted bioRxiv a license to display the preprint in perpetuity. It is made available under aCC-BY-NC-ND 4.0 International license.

\section{Figure 5: CRISPR screens elucidate distinct mechanisms of regulatory driver function.}

A. CRISPR dependency scores of distal. promoter, and coding genes

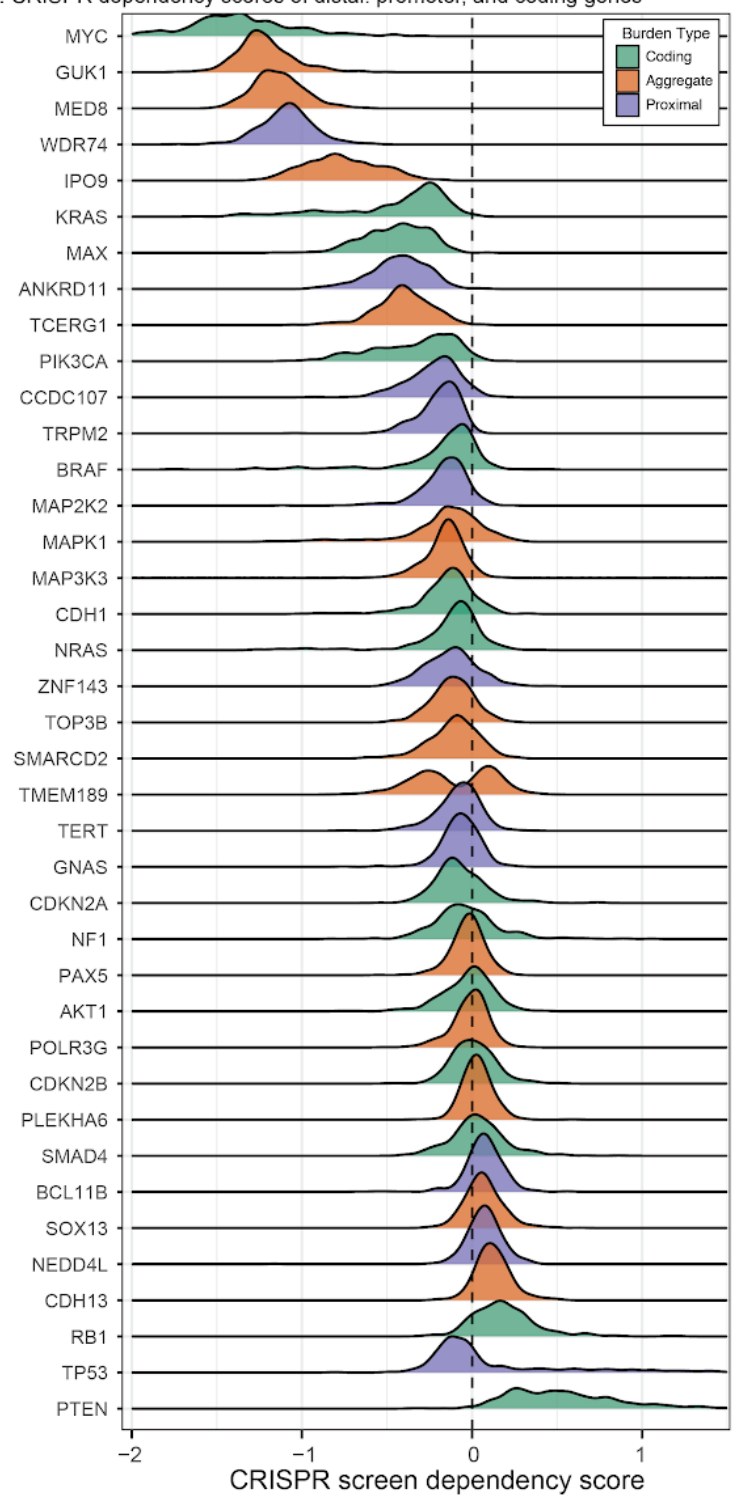

C. Cancer type specificity of putative regulatory driver genes

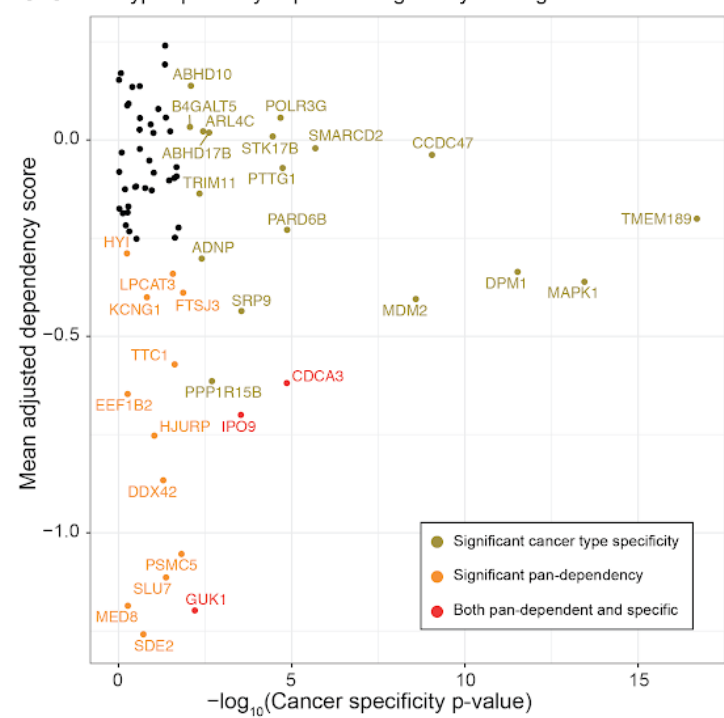

D. Comparison between IPO9 and known coding genes

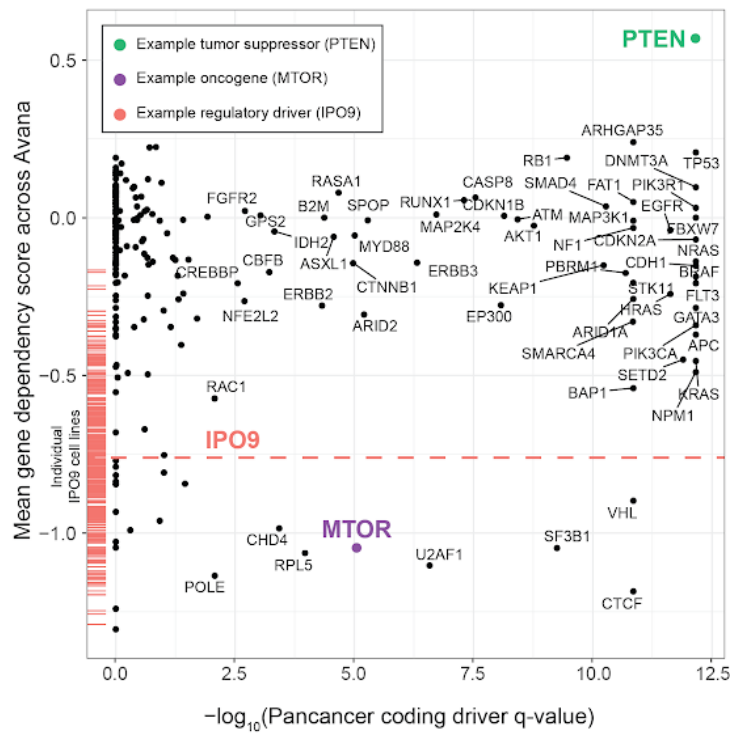

B. Gene dependency distributions for mutated gene classes

E. Positive growth effect at OXNAD1 locus

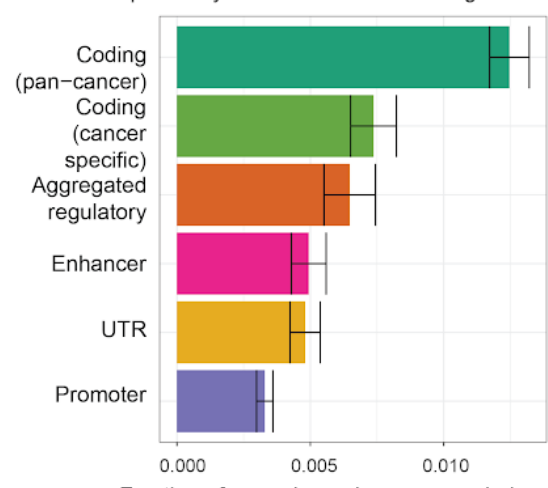

Fraction of gene dependency scores below -0.5

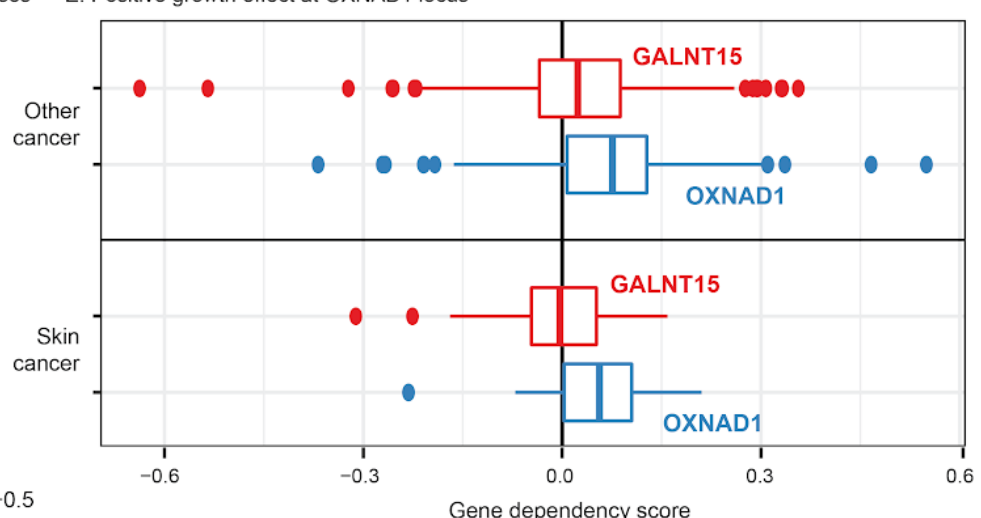


A. CRISPR dependency scores of distal, promoter, and coding drivers. Effect on CRISPR growth of known promoters and coding drivers versus novel regulatory drivers. Each distribution is the effects observed across cell lines. Essential gene knockouts have a median dependency score of -1.0 , while non-essential gene knockouts have a median dependency score of 0 .

B. Gene dependency distributions for mutated gene classes. Across all associated genes, the knockout dependency scores of regulatory, promoter, and coding associated variants relative to the whole genome background. The fraction of gene effects below -0.5 (indicating substantial deleterious effect on proliferation) (Meyers et al., 2017)) are tallied across all genes in the given set. Coding data are from TCGA (Lawrence et al., 2014) and non-aggregate non-coding data are from PCAWG (Rheinbay et al., 2020).

C. Cancer type specificity of putative regulatory driver genes. Each gene was evaluated for cancer type specificity using an F-test (Methods) and the resulting estimates were used to separate genes into those with significant specificity (gold), non-zero aggregate essentiality (orange), both (red), or neither (black).

D. Comparison between IPO9 and known coding genes. Comparison of coding versus noncoding effects in the Achilles screens. Each dot represents a significant pan- or single-cancer association from Lawrence et al (2014). The red dashed line and bars are the mean estimate and individual estimates of effect for IPO9.

E. Positive growth effect at OXNAD1 locus. Both GALNT15 and OXNAD1 have regulatory regions overburdened with mutations, but CRISPR/Cas9 screens reveal a significantly larger positive dependency score for OXNAD1 compared to GALNT15 in melanoma and other cell lines. 


\section{Figure 6: Regulatory and functional characterization of IPO9 using CRISPR screen data.}

A. CRISPR gene effects at the IPO9 locus B. Strategy for estimating shared effects with IPO9 across cell lines
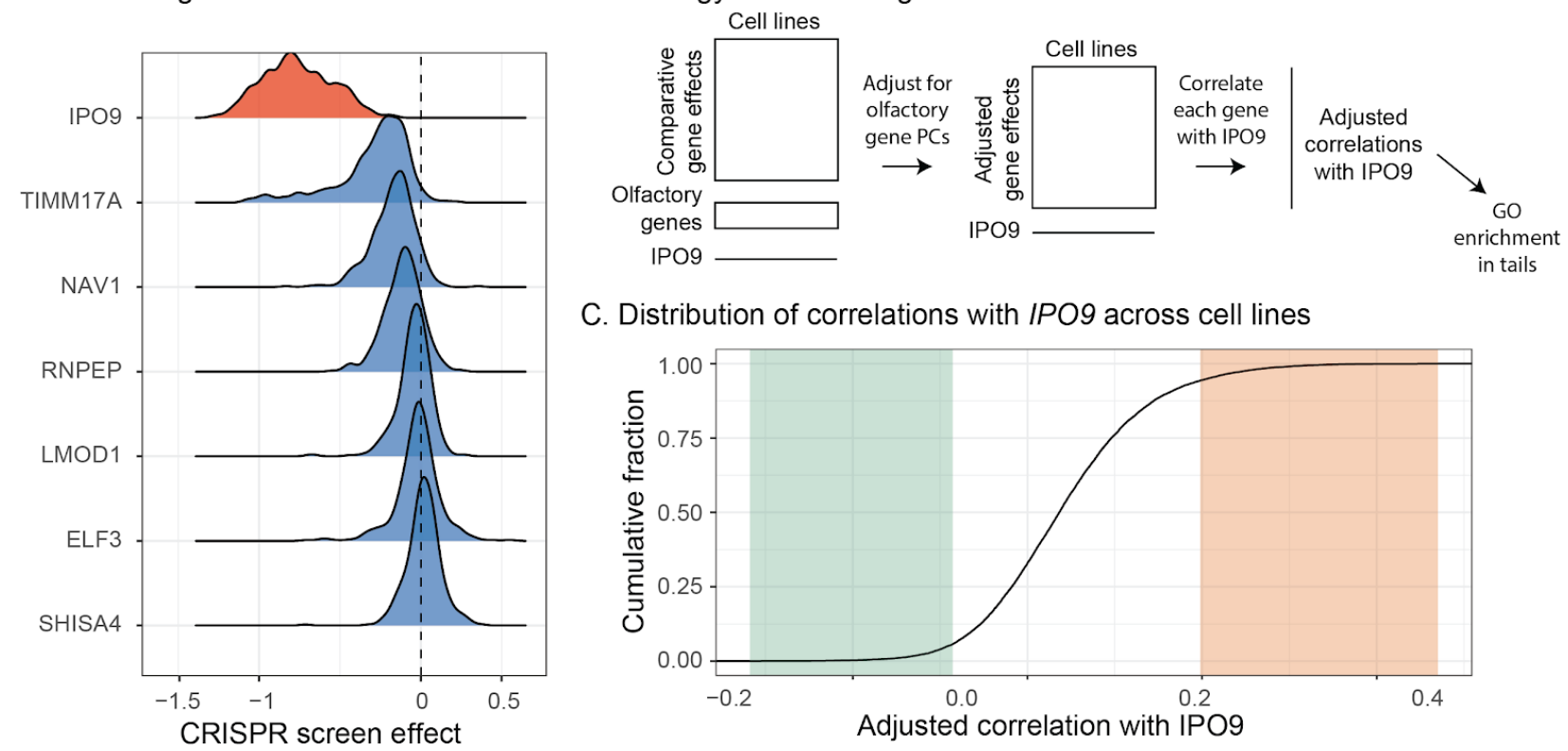

C. Distribution of correlations with IPO9 across cell lines

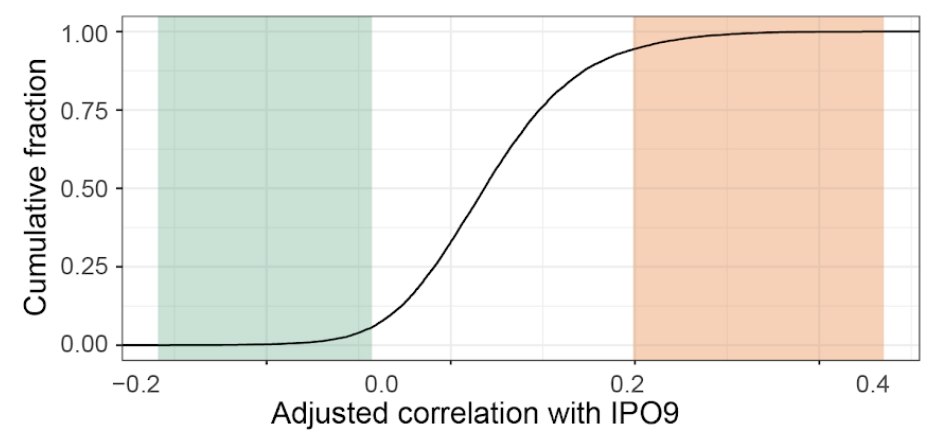

1132 D. GO enrichment for splicing and RNA binding in genes correlated with IPO9

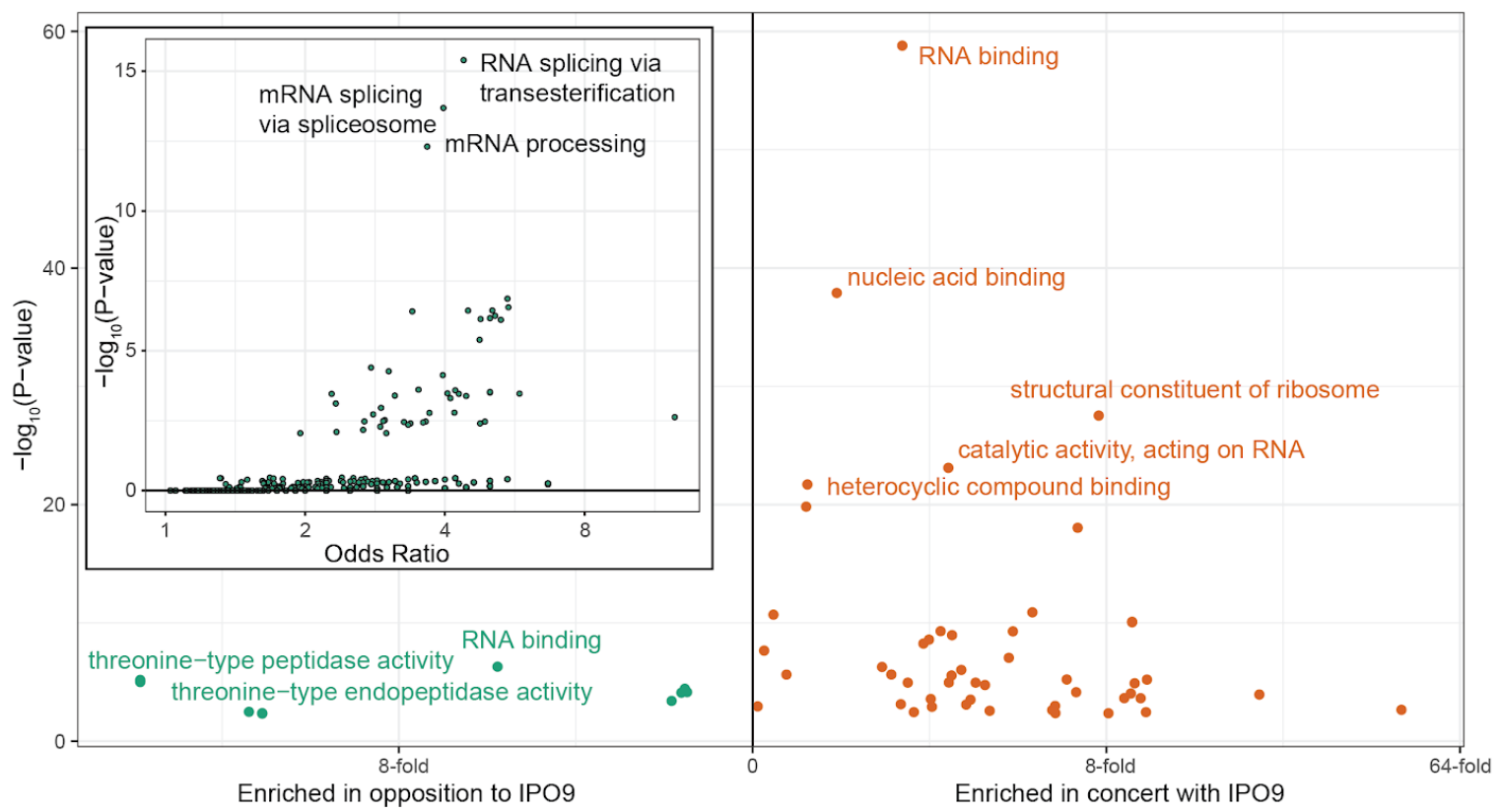

A. CRISPR gene effects at the IPO9 locus. Overall distribution of growth effect of IPO9 versus all other genes at the locus in CRISPR/Cas9 gene knockouts across cancer cell lines (Meyers et al., 2017).

B. Strategy for estimating shared effects with IPO9 across cell lines. Overall schematic of our method for estimating shared effects across cell lines, similar to previous designs (Boyle et al., 2018). 
1139

1140

1141

1142

1143

1144

1145

1146

1147

1148
C. Distribution of correlations with IPO9 across cell lines. The observed distribution of batch-corrected correlations between IPO9 and each other genes across Avana and cell lines. Green, negatively correlated genes and orange, positively correlated genes.

D. GO enrichment for splicing and RNA binding in genes correlated with IPO9. Volcano plots of enrichment for the ranked gene list correlation with IPO9, showing a consistent signal of RNA processing. [inset] Volcano plot of enrichment within tail (correlation threshold 0.3), illustrating a substantial enrichment for RNA splicing related genes. Green, negatively correlated genes and orange, positively correlated genes. 
bioRxiv preprint doi: https://doi.org/10.1101/2020.08.21.239954; this version posted August 31, 2020. The copyright holder for this preprint (which was not certified by peer review) is the author/funder, who has granted bioRxiv a license to display the preprint in perpetuity. It is made available under aCC-BY-NC-ND 4.0 International license.

\section{Figure 7: Recurrently mutated genes are associated with clinical outcome.}

A. Hazard plot by cancer type (The Cancer Genome Atlas)

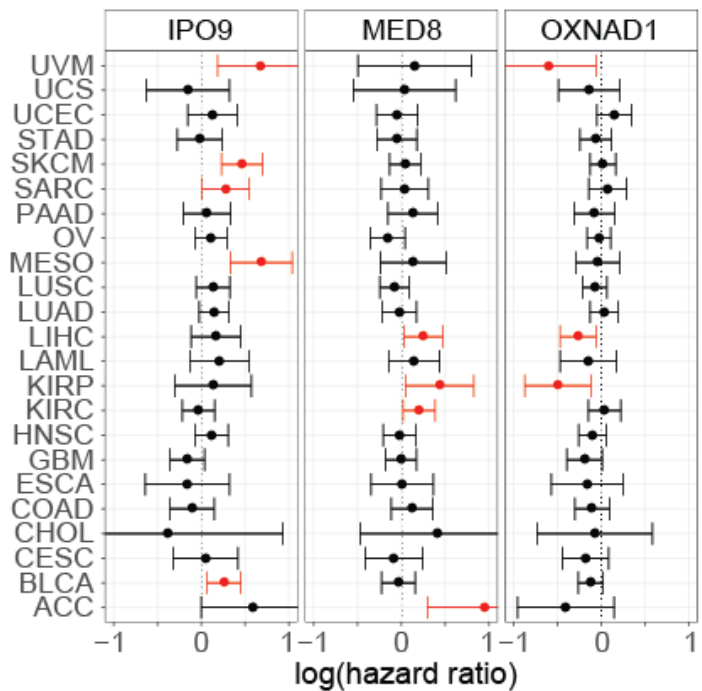

C. OXNAD1 relapse free survival in uveal melanoma

1150

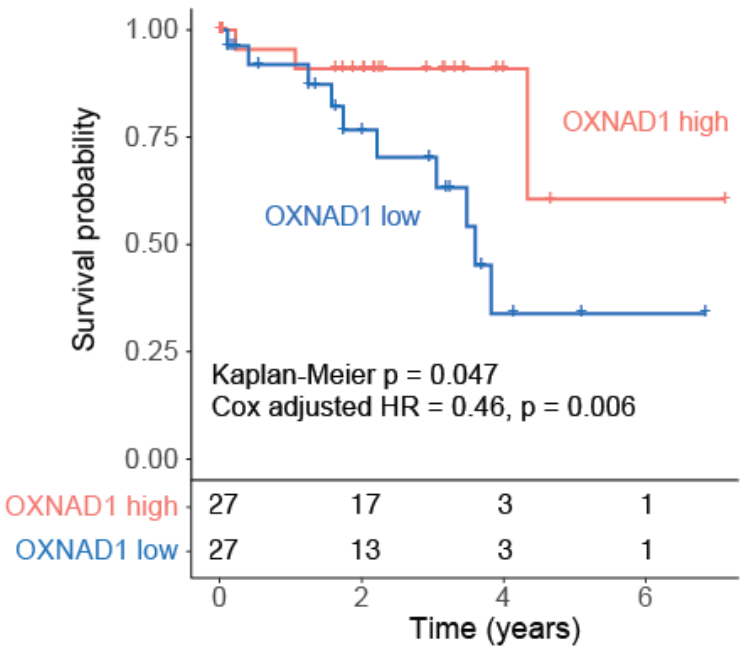

E. IPO9 survival in luminal breast cancer (METABRIC)

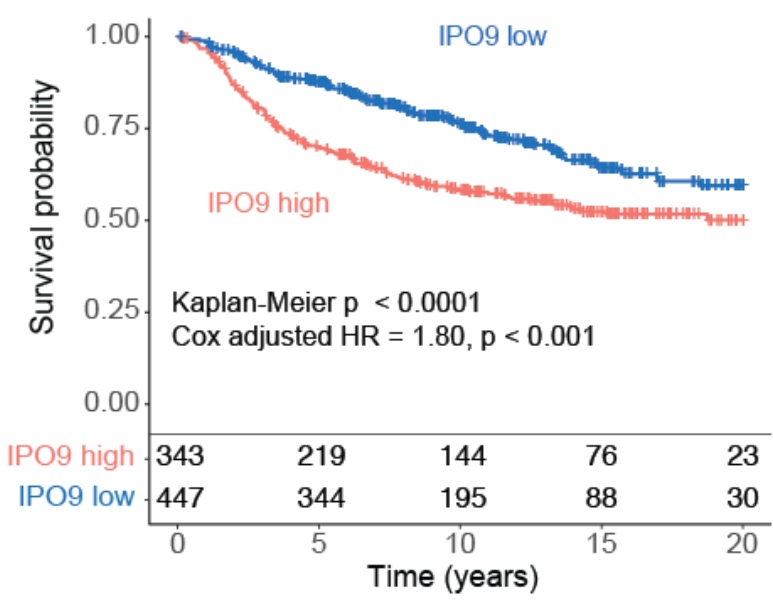

B. IPO9 relapse free survival in melanoma

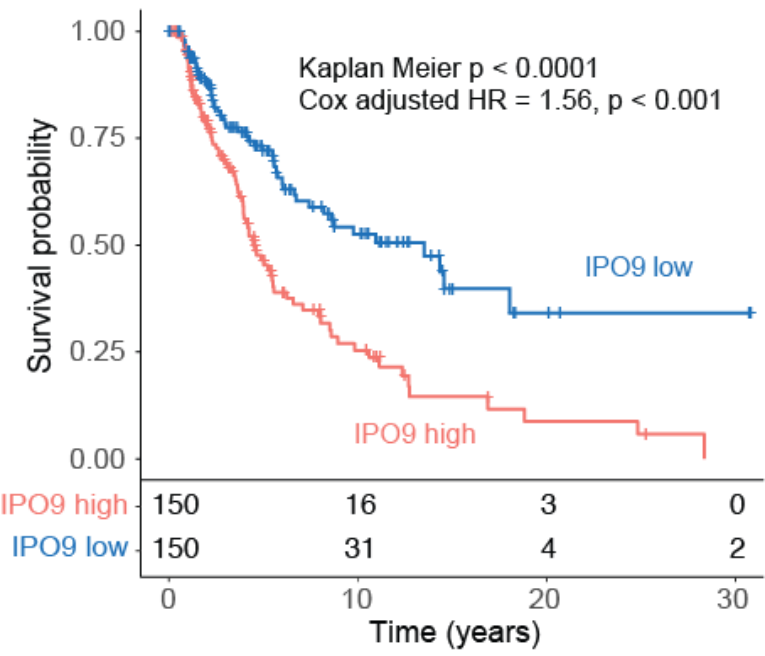

D. MED8 relapse free survival in kidney papillary carcinoma

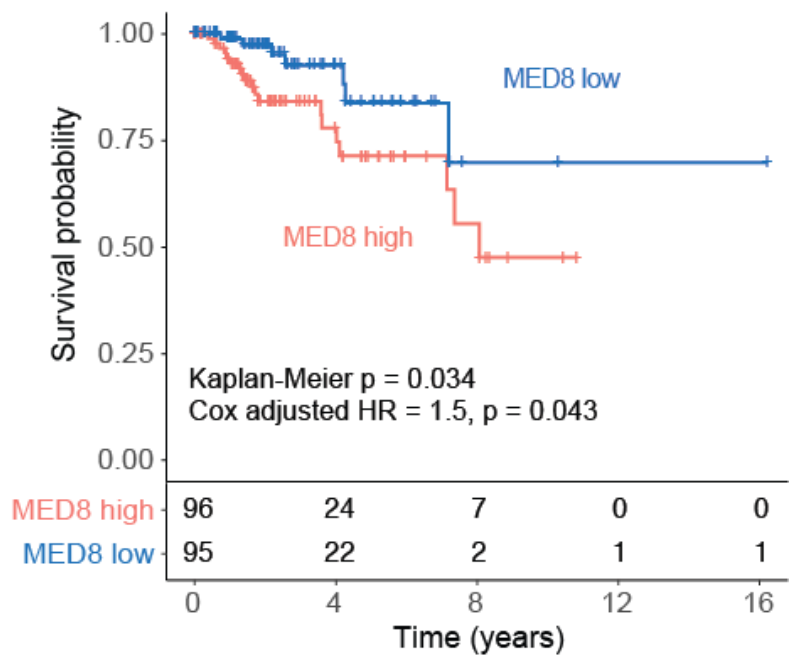

F. Luminal breast cancer coding and aggregated drivers

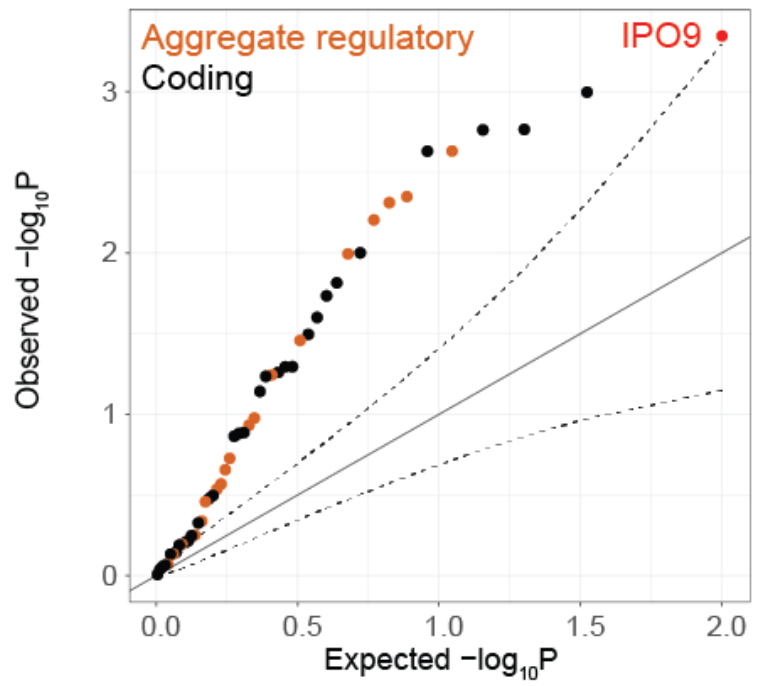


A. Hazard plot by cancer type. Forest plot for IPO9, MED8, and OXNAD1 from expression and relapse-free survival Cox proportional hazards in TCGA across 23 well-powered cancer types (breast was excluded due to limited followup duration in TCGA). Data for GUK1 and PLEKHA6 are reported in Supplemental Figure 6.

B. IPO9 relapse free survival in melanoma (TCGA). Kaplan-Meier analysis of the association between IPO9 expression and relapse free survival in the TCGA melanoma cohort. Cox Proportional Hazards Ratios are also reported. Corresponding forest plot in Supplemental Figure 7, and uncensored counts presented below the axis for each timepoint.

C. OXNAD1 relapse free survival associations in uveal melanoma (TCGA).

Kaplan-Meier analysis of the association between OXNAD1 expression and relapse free survival in the TCGA uveal melanoma cohort. Cox Proportional Hazards Ratios are also reported. Corresponding forest plot in Supplemental Figure 7, and uncensored counts presented below the axis for each timepoint.

D. MED8 relapse free survival associations in kidney papillary carcinoma (TCGA). Kaplan-Meier analysis of the association between MED8 expression and relapse free survival in the TCGA ukidney papillary carcinoma cohort. Cox Proportional Hazards Ratios are also reported. Corresponding forest plot in Supplemental Figure 7, and uncensored counts presented below the axis for each timepoint.

E. IPO9 relapse free survival associations in luminal breast cancer (METABRIC). Kaplan-Meier analysis of the association between IPO9 expression and relapse free survival in the METABRIC breast cancer cohort. Cox Proportional Hazards Ratios are also reported. Corresponding forest plot and forest plot for all cancers in Supplemental Figure 7, and uncensored counts presented below the axis for each timepoint.

F. Luminal breast cancer coding and aggregated drivers. Quantile-quantile plot of gene expression-survival associations based on disease-free survival in luminal cases in METABRIC. The distribution covers recurrently altered coding variants from TCGA or aggregated regulatory genes from our study $(n=50)$, revealing enrichment for survival associations. Corresponding plot for all tumors in Supplemental Figure 7. 